\title{
A Simple glycosylation of allyl glycosides
}

Pengfei Wang*, Pranab Haldar, Yun Wang and Huayou Hu

Department of Chemistry

University of Alabama at Birmingham

Birmingham, Alabama 35294

\section{SUPPORTING MATERIALS}

\section{Contents}

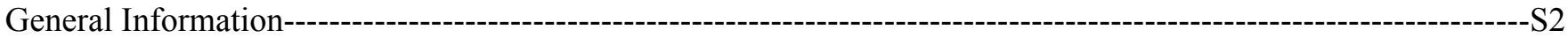

Spectroscopic Data of $11 \beta, 11 \alpha, 15 \beta, 15 \alpha, 18 \beta, 18 \alpha, 19 \beta, 19 \alpha, 20 \beta, 20 \alpha, 21 \beta \beta, 21 \alpha \beta, 21 \beta \alpha, 22 \beta, 22 \alpha$, and $23 \beta$

NMR spectra of $11 \beta, 11 \alpha, 14 \beta, 14 \alpha, 15 \beta, 15 \alpha, 18 \beta, 18 \alpha, 19 \beta, 19 \alpha, 20 \beta, 20 \alpha, 21 \beta \beta, 21 \alpha \beta, 21 \beta \alpha, 22 \beta, 22 \alpha$, and 23 $\beta$ 
General Procedures. Glycosylation reactions were performed in Schlenk (Kjeldahl shape) flasks under a positive pressure of argon. The glycosylation partners were dried by azeotropic removal of water with toluene prior to initiation of the glycosylation reactions. Organic solutions were concentrated by rotary evaporation at ca. 12 Torr. Flash column chromatography was performed employing 230-400 mesh silica gel. Thin-layer chromatography (analytical and preparative) was performed using glass plates pre-coated to a depth of $0.25 \mathrm{~mm}$ with $230-400$ mesh silica gel impregnated with a fluorescent indicator $(254 \mathrm{~nm})$.

Materials. Tetrahydrofuran, toluene, acetonitrile were distilled from appropriate drying reagents under a nitrogen atmosphere at 760 torr. Other chemicals were obtained from commercial vendors and used without further purification.

Instrumentation. Infrared (IR) data are presented as frequency of absorption $\left(\mathrm{cm}^{-1}\right)$. Proton and carbon-13 nuclear magnetic resonance $\left({ }^{1} \mathrm{H}\right.$ NMR or ${ }^{13} \mathrm{C}$ NMR) spectra were recorded on a $300 \mathrm{MHz}$ and a $400 \mathrm{MHz} \mathrm{NMR}$ spectrometer; chemical shifts are expressed in parts per million $(\delta$ scale) downfield from tetramethylsilane and are referenced to residual protium in the NMR solvent $(\mathrm{CHCl3}: \delta$ 7.26). Data are presented as follows: chemical shift, multiplicity $(\mathrm{s}=$ singlet, $\mathrm{d}=$ doublet, $\mathrm{t}=$ triplet, $\mathrm{q}=$ quartet, $\mathrm{m}=$ multiplet and/or multiple resonances), coupling constant in Hertz (Hz), integration.

\section{Spectroscopic Data}

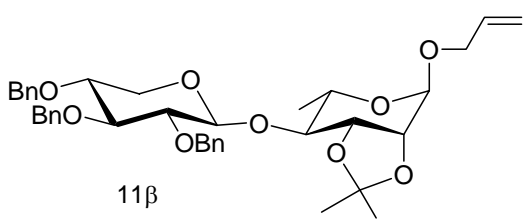

Disaccharide 11 $\beta$. Rf 0.37 (benzene/ethyl acetate 19:1); ${ }^{1} \mathrm{H}$ NMR (400 MHz, $\left.\mathrm{CDCl}_{3}\right) \delta$ 7.40-7.20 (m, $\left.15 \mathrm{H}\right)$, $5.90(\mathrm{~m}, 1 \mathrm{H}), 5.32(\mathrm{dq}, \mathrm{J}=17.2,1.6 \mathrm{~Hz}, 1 \mathrm{H}), 5.22(\mathrm{ddt}, \mathrm{J}=10.3,1.7,1.2 \mathrm{~Hz}, 1 \mathrm{H}), 5.02(\mathrm{~s}, 1 \mathrm{H}), 4.91(\mathrm{~d}, \mathrm{~J}$ $=7.7 \mathrm{~Hz}, 1 \mathrm{H}), 4.88(\underline{\mathrm{AB}}, \mathrm{J}=11.1 \mathrm{~Hz}, 1 \mathrm{H}), 4.90-4.80(\mathrm{AB}, \mathrm{J}=11.0 \mathrm{~Hz}, 2 \mathrm{H}), 4.71(\underline{\mathrm{AB}}, \mathrm{J}=11.7 \mathrm{~Hz}, 1 \mathrm{H})$, $4.69(\mathrm{AB}, \mathrm{J}=11.1 \mathrm{~Hz}, 1 \mathrm{H}), 4.63(\mathrm{AB}, \mathrm{J}=11.7 \mathrm{~Hz}, 1 \mathrm{H}), 4.28-4.20(\mathrm{~m}, 1 \mathrm{H}), 4.19(\underline{\mathrm{ABMX}} 2, \mathrm{~J}=12.8,5.3$, $1.4 \mathrm{~Hz}, 1 \mathrm{H}), 4.13(\mathrm{dd}, \mathrm{J}=5,6,0.6 \mathrm{~Hz}, 1 \mathrm{H}), 4.00\left(\mathrm{ABMX}_{2}, \mathrm{~J}=12.8,6.2,1.3 \mathrm{~Hz}, 1 \mathrm{H}\right), 3.98-3.91(\mathrm{~m}, 1 \mathrm{H})$, 3.70-3.57 (m, $4 \mathrm{H}), 3.34-3.27(\mathrm{~m}, 1 \mathrm{H}), 3.25-3.15(\mathrm{~m}, 1 \mathrm{H}), 1.51(\mathrm{~s}, 3 \mathrm{H}), 1.34(\mathrm{~s}, 3 \mathrm{H}), 1.27(\mathrm{~d}, \mathrm{~J}=5.7 \mathrm{~Hz}, 3$ $\mathrm{H}) ;{ }^{13} \mathrm{C}$ NMR $(101 \mathrm{MHz}, \mathrm{CDCl} 3) \delta 138.7,138.6,138.2,133.6,128.4,128.3,128.3,128.1,127.9,127.8$, $127.5,117.8,109.2,101.9,96.09,8.85,82.0,78.2,78.0,77.9,76.0,75.6,74.8,73.2,68.0,64.2,63.77,27.8$, 26.3, 17.6; FTIR (neat) $\mathrm{cm}^{-1}$ 3030, 2983, 2904, 1496, 1455, 1372, 1086; HRMS (ESI) m/z: Calcd for $\mathrm{C}_{38} \mathrm{H}_{46} \mathrm{O}_{9} \mathrm{Na}(\mathrm{M}+\mathrm{Na}) 669.3040$, found 669.3029 .

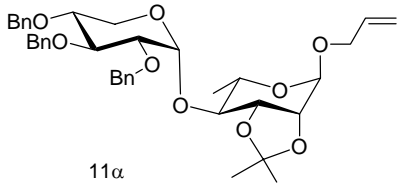

Disaccharide 11 $\alpha$. Rf 0.30 (benzene/ethyl acetate 19:1); ${ }^{1} \mathrm{H}$ NMR $\left(400 \mathrm{MHz}, \mathrm{CDCl}_{3}\right) \delta 7.45-7.20(\mathrm{~m}, 15 \mathrm{H})$, $5.90(\mathrm{~m}, 1 \mathrm{H}), 5.30(\mathrm{dq}, \mathrm{J}=17.2,1.6 \mathrm{~Hz}, 1 \mathrm{H}), 5.21(\mathrm{ddt}, \mathrm{J}=10.3,1.6,1.2 \mathrm{~Hz}, 1 \mathrm{H}), 5.01(\mathrm{~s}, 1 \mathrm{H}), 4.90(\mathrm{~s}, 2$ $\mathrm{H}), 3.88(\mathrm{~d}, \mathrm{~J}=3.7 \mathrm{~Hz}, 1 \mathrm{H}), 4.81(\underline{\mathrm{AB}}, \mathrm{J}=11.7 \mathrm{~Hz}, 1 \mathrm{H}), 4.73(\underline{\mathrm{AB}}, \mathrm{J}=11.6 \mathrm{~Hz}, 1 \mathrm{H}), 4.69$ (Aㅁ, J =11.6 $\mathrm{Hz}, 1 \mathrm{H}), 4.65(\mathrm{AB}, \mathrm{J}=11.6 \mathrm{~Hz}, 1 \mathrm{H}), 4.22-4.11(\mathrm{~m}, 3 \mathrm{H}), 4.01-3.86(\mathrm{~m}, 3 \mathrm{H}), 3.86-3.75(\mathrm{~m}, 1 \mathrm{H}), 3.62-3.54$ $(\mathrm{m}, 2 \mathrm{H}), 3.46(\mathrm{dd}, \mathrm{J}=9.7,3.7 \mathrm{~Hz}, 1 \mathrm{H}), 3.37$ (dd, J = 10.1, $7.4 \mathrm{~Hz}, 1 \mathrm{H}), 1.49$ (s, $3 \mathrm{H}), 1.34$ (s, $3 \mathrm{H}), 1.31$ (d, $\mathrm{J}=6.4 \mathrm{~Hz}, 3 \mathrm{H}) ;{ }^{13} \mathrm{C} \mathrm{NMR}(101 \mathrm{MHz}, \mathrm{CDCl} 3) \delta 138.8,138.3,138.0,133.6,128.4,128.3,128.2,127.9$, $127.9,127.8,127.7,127.5,117.8,109.0,98.0,95.9,81.4,80.4,79.7,78.4,76.1,75.6,74.3,73.4,67.8,65.0$, 60.4, 27.8, 26.5, 17.4; FTIR (neat) $\mathrm{cm}^{-1}$ 2904, 1496, 1369, 1086, 1027; HRMS (ESI) m/z: Calcd for $\mathrm{C}_{38} \mathrm{H}_{46} \mathrm{O}_{9} \mathrm{Na}(\mathrm{M}+\mathrm{Na}) 669.3040$, found 669.3049. 


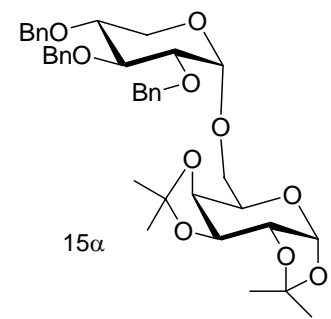

Disaccharide 15 $\alpha .{ }^{1}$ Rf 0.47 (benzene/ethyl acetate 9:1); ${ }^{1} \mathrm{H}$ NMR $\left(400 \mathrm{MHz}, \mathrm{CDCl}_{3}\right) \delta$ 7.40-7.20 (m, $\left.15 \mathrm{H}\right), 5.53(\mathrm{~d}, \mathrm{~J}$ $=5.1 \mathrm{~Hz}, 1 \mathrm{H}), 4.92(\underline{\mathrm{AB}}, \mathrm{J}=10.9 \mathrm{~Hz}, 1 \mathrm{H}), 4.89(\mathrm{~d}, \mathrm{~J}=3.7 \mathrm{~Hz}, 1 \mathrm{H}), 4.86(\mathrm{AB}, \mathrm{J}=10.9 \mathrm{~Hz}, 1 \mathrm{H}), 4.75-4.68(\mathrm{~m}, 3 \mathrm{H})$, 4.65-4.58 (m, $2 \mathrm{H}), 4.37$ (dd, J = 7.9, $1.8 \mathrm{~Hz}, 1 \mathrm{H}), 4.32(\mathrm{dd}, \mathrm{J}=5.0,2.4 \mathrm{~Hz}, 1 \mathrm{H}), 4.04$ (td, J = 6.1, $0.5 \mathrm{~Hz}, 1 \mathrm{H}), 3.90$ $(\mathrm{t}, \mathrm{J}=8.7 \mathrm{~Hz}, 1 \mathrm{H}), 3.77(\underline{\mathrm{ABX}}, \mathrm{J}=10.2,6.0 \mathrm{~Hz}, 1 \mathrm{H}), 3.71(\mathrm{ABX}, \mathrm{J}=10.2,7.8 \mathrm{~Hz}, 1 \mathrm{H}), 3.65-3.50(\mathrm{~m}, 3 \mathrm{H}), 3.46$ $(\mathrm{dd}, \mathrm{J}=9.5,3.6 \mathrm{~Hz}, 1 \mathrm{H}), 1.55(\mathrm{~s}, 3 \mathrm{H}), 1.45(\mathrm{~s}, 3 \mathrm{H}), 1.33(\mathrm{~s}, 3 \mathrm{H}), 1.31(\mathrm{~s}, 3 \mathrm{H}) ;{ }^{13} \mathrm{C} \mathrm{NMR}\left(101 \mathrm{MHz}, \mathrm{CDCl}_{3}\right) \delta$ 139.2, 138.6, 138.6, 128.6, 128.5, 128.2, 128.0, 128.0, 127.9, 127.7, 109.4, 108.8, 97.4, 96.5, 81.5, 79.8, 78.3, 75.9, 73.7, 72.8, 71.0, 70.9, 70.8, 66.5, 66.0, 60.3, 29.9, 26.3, 25.2, 24.8; FTIR (neat) $\mathrm{cm}^{-1} 2932,1379,1072$; HRMS (ESI) $\mathrm{m} / \mathrm{z}$ : Calcd for $\mathrm{C}_{38} \mathrm{H}_{46} \mathrm{O}_{10} \mathrm{Na}(\mathrm{M}+\mathrm{Na}) 685.2989$, found 685.2975.

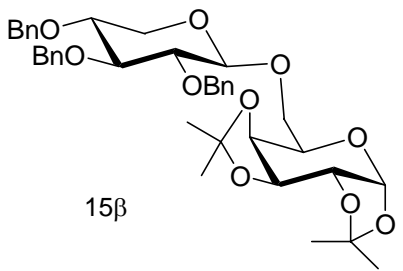

Disaccharide 15 $\beta .{ }^{1} \mathrm{Rf} 0.37$ (benzene/ethyl acetate 9:1); ${ }^{1} \mathrm{H}$ NMR $\left(400 \mathrm{MHz}, \mathrm{CDCl}_{3}\right) \delta$ 7.45-7.20 (m, $\left.15 \mathrm{H}\right), 5.58(\mathrm{~d}, \mathrm{~J}$ $=5.0 \mathrm{~Hz}), 4.89(\underline{\mathrm{AB}}, \mathrm{J}=11.1 \mathrm{~Hz}, 1 \mathrm{H}), 4.88(\underline{\mathrm{AB}}, \mathrm{J}=11.0 \mathrm{~Hz}, 1 \mathrm{H}), 4.82(\mathrm{AB}, \mathrm{J}=11.0 \mathrm{~Hz}, 1 \mathrm{H}), 4.72(\underline{\mathrm{AB}}, \mathrm{J}=11.6$ $\mathrm{Hz}, 1 \mathrm{H}), 4.70(\mathrm{AB}, \mathrm{J}=11.1 \mathrm{~Hz}, 1 \mathrm{H}), 4.61(\mathrm{AB}, \mathrm{J}=11.8 \mathrm{~Hz}, 1 \mathrm{H}), 4.59(\mathrm{dd}, \mathrm{J}=7.9,2.4 \mathrm{~Hz}, 1 \mathrm{H}), 4.40(\mathrm{~d}, \mathrm{~J}=7.6 \mathrm{~Hz}$, $1 \mathrm{H}), 4.32(\mathrm{dd}, \mathrm{J}=5.0,2.4 \mathrm{~Hz}, 1 \mathrm{H}), 4.22(\mathrm{dd}, \mathrm{J}=7.9,1.7 \mathrm{~Hz}, 1 \mathrm{H}), 4.08-4.02(\mathrm{~m}, 2 \mathrm{H}), 3.90(\mathrm{ABX}, \mathrm{J}=11.7,5.0 \mathrm{~Hz}, 1$ H), 3.77 (dd, J = 12.0, $8.6 \mathrm{~Hz}, 1 \mathrm{H}), 3.62-3.53(\mathrm{~m}, 2 \mathrm{H}), 3.38$ (t, J = 7.7 Hz, $1 \mathrm{H}), 3.19$ (dd, J = 10.8, 9.2 Hz, $1 \mathrm{H}), 1.49$ $(\mathrm{s}, 3 \mathrm{H}), 1.45(\mathrm{~s}, 3 \mathrm{H}), 1.32(\mathrm{~s}, 3 \mathrm{H}), 1.31(\mathrm{~s}, 3 \mathrm{H}) ;{ }^{13} \mathrm{C} \mathrm{NMR}\left(101 \mathrm{MHz}, \mathrm{CDCl}_{3}\right) \delta$ 138.7, 138.6, 138.1, 128.4, 128.3, 128.2, 127.9, 127.8, 127.7, 127.5, 127.5, 109.4, 108.5, 104.9, 96.3, 83.6, 81.4, 77.7, 75.6, 74.5, 73.4, 71.3, 70.7, 70.4, 69.4, 67.1, 63.9; FTIR (neat) $\mathrm{cm}^{-1}$ 3062, 3031, 2986, 2903, 1719, 1455, 1379, 1256, 1212, 1172, 1073; HRMS (ESI) $\mathrm{m} / \mathrm{z}$ : Calcd for $\mathrm{C}_{38} \mathrm{H}_{46} \mathrm{O}_{10} \mathrm{Na}(\mathrm{M}+\mathrm{Na})$ 685.2989, found 685.2992.

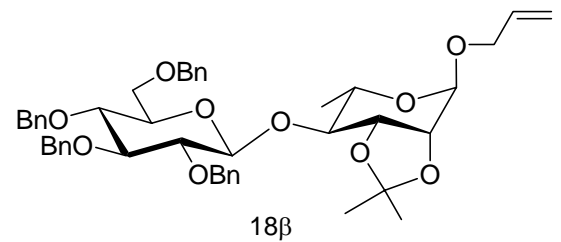

Disaccharide 18 $\beta{ }^{2}$ Rf 0.30 (petroleum ether/ethyl acetate 7:1); ${ }^{1} \mathrm{H}$ NMR $\left(400 \mathrm{MHz}, \mathrm{CDCl}_{3}\right) \delta 7.40-7.10(\mathrm{~m}, 20 \mathrm{H})$, $5.94(\mathrm{~m}, 1 \mathrm{H}), 5.33(\mathrm{dq}, \mathrm{J}=17.2,1.6 \mathrm{~Hz}, 1 \mathrm{H}), 5.24(\mathrm{ddt}, \mathrm{J}=10.4,1.6,1.2 \mathrm{~Hz}, 1 \mathrm{H}), 5.03(\mathrm{~s}, 1 \mathrm{H}), 4.97-4.91(\mathrm{~m}, 3 \mathrm{H})$, $4.83(\underline{\mathrm{AB}}, \mathrm{J}=10.9 \mathrm{~Hz}, 1 \mathrm{H}), 4.79(\mathrm{AB}, \mathrm{J}=11.0 \mathrm{~Hz}, 1 \mathrm{H}), 4.71(\mathrm{AB}, \mathrm{J}=11.1 \mathrm{~Hz}, 1 \mathrm{H}), 4.62(\underline{\mathrm{AB}}, \mathrm{J}=12.3 \mathrm{~Hz}, 1 \mathrm{H})$, $4.59(\mathrm{~A} \underline{B}, \mathrm{~J}=11.0 \mathrm{~Hz}, 1 \mathrm{H}), 4.55(\mathrm{AB}, \mathrm{J}=12.3 \mathrm{~Hz}, 1 \mathrm{H}), 4.24(\mathrm{~m}, 1 \mathrm{H}), 4.21\left(\mathrm{ABMX}_{2}, \mathrm{~J}=12.8,5.3,1.3 \mathrm{~Hz}, 1 \mathrm{H}\right)$, $4.14(\mathrm{~d}, \mathrm{~J}=5.6 \mathrm{~Hz}, 1 \mathrm{H}), 4.02\left(\mathrm{ABMX}_{2}, \mathrm{~J}=12.8,6.2,1.3 \mathrm{~Hz}, 1 \mathrm{H}\right), 3.75-3.62(\mathrm{~m}, 6 \mathrm{H}), 3.44-3.38(\mathrm{~m}, 2 \mathrm{H}), 1.47(\mathrm{~s}, 3$ $\mathrm{H}), 1.34(\mathrm{~d}, \mathrm{~J}=5.9 \mathrm{~Hz}, 3 \mathrm{H}), 1.33(\mathrm{~s}, 3 \mathrm{H}) ;{ }^{13} \mathrm{C} \mathrm{NMR}\left(101 \mathrm{MHz}, \mathrm{CDCl}_{3}\right) \delta 138.7,138.6,138.2,133.6,128.3,128.3$, $128.3,128.2,127.9,127.8,127.7,127.6,127.5,117.8,109.2,101.6,96.1,84.8,82.4,78.3,78.2,77.8,76.0,74.8,74.7$, 73.4, 68.6, 68.0, 64.4, 27.8, 26.3, 17.7; FTIR (neat) $\mathrm{cm}^{-1}$ 3063, 3030, 2984, 2907, 1496, 1370, 1077; HRMS (ESI) $\mathrm{m} / \mathrm{z}$ : Calcd for $\mathrm{C}_{46} \mathrm{H}_{54} \mathrm{O}_{10} \mathrm{Na}(\mathrm{M}+\mathrm{Na}) 789.3615$, found 789.3644 .

\footnotetext{
${ }^{1}$ Known compounds, see Chen, Q; Kong, F. Carbohydr. Res. 1995, 272, 149.

${ }^{2}$ Known compounds, see: Mulard, L. A.; Costachel, C.; Sansonetti, P. J. J. Carbohydr. Chem. 2000, $19,849$.
} 


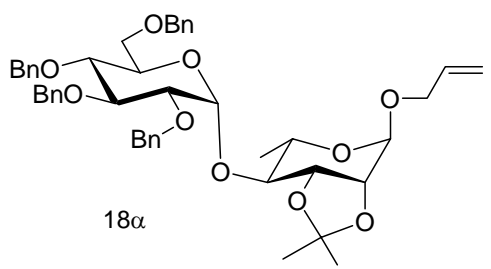

Disaccharide 18 $\alpha .{ }^{2} \operatorname{Rf} 0.20$ (petroleum ether/ethyl acetate 7:1); ${ }^{1} \mathrm{H}$ NMR $\left(400 \mathrm{MHz}, \mathrm{CDCl}_{3}\right) \delta$ 7.40-7.20 (m, $\left.18 \mathrm{H}\right)$, 7.20-7.10 (m, $2 \mathrm{H}), 5.88(\mathrm{~m}, 1 \mathrm{H}), 5.29(\mathrm{dq}, \mathrm{J}=17.2,1.5 \mathrm{~Hz}, 1 \mathrm{H}), 5.21(\mathrm{dq}, \mathrm{J}=10.3,1.2 \mathrm{~Hz}, 1 \mathrm{H}), 4.98(\mathrm{~s}, 1 \mathrm{H}), 4.97$ $(\underline{\mathrm{AB}}, \mathrm{J}=10.9 \mathrm{~Hz}, 1 \mathrm{H}), 4.96(\mathrm{~d}, \mathrm{~J}=3.7 \mathrm{~Hz}, 1 \mathrm{H}), 4.88(\mathrm{AB}, \mathrm{J}=10.9 \mathrm{~Hz}, 1 \mathrm{H}), 4.83(\underline{\mathrm{AB}}, \mathrm{J}=11.0 \mathrm{~Hz}, 1 \mathrm{H}), 4.79(\underline{\mathrm{AB}}$, $\mathrm{J}=11.6 \mathrm{~Hz}, 1 \mathrm{H}), 4.71(\mathrm{AB}, \mathrm{J}=11.6 \mathrm{~Hz}, 1 \mathrm{H}), 4.62(\underline{\mathrm{AB}}, \mathrm{J}=12.1 \mathrm{~Hz}, 1 \mathrm{H}), 4.52(\mathrm{~A} \underline{\mathrm{B}}, \mathrm{J}=10.9 \mathrm{~Hz}, 1 \mathrm{H}), 4.49(\mathrm{~A} \underline{\mathrm{B}}, \mathrm{J}$ $=12.1 \mathrm{~Hz}, 1 \mathrm{H}), 4.16-4.06(\mathrm{~m}, 4 \mathrm{H}), 4.01-2.92(\mathrm{~m}, 2 \mathrm{H}), 3.83-3.76(\mathrm{~m}, 3 \mathrm{H}), 3.63(\mathrm{dd}, \mathrm{J}=10.5,2.0 \mathrm{~Hz}, 1 \mathrm{H}), 3.60(\mathrm{dd}$, $\mathrm{J}=9.8,3.6 \mathrm{~Hz}, 1 \mathrm{H}), 3.33(\mathrm{dd}, \mathrm{J}=10.2,7.0 \mathrm{~Hz}, 1 \mathrm{H}), 1.42(\mathrm{~s}, 3 \mathrm{H}), 1.30(\mathrm{~d}, \mathrm{~J}=6.3 \mathrm{~Hz}, 3 \mathrm{H}), 1.24(\mathrm{~s}, 3 \mathrm{H}) ;{ }^{13} \mathrm{C} \mathrm{NMR}$ $\left(101 \mathrm{MHz}, \mathrm{CDCl}_{3}\right) \delta 138.8,138.3,138.0,137.8,133.5,128.4,128.4,128.3,128.0,127.9,127.8,127.7,127.6,117.8$, 108.9, 98.3, 95.9, 82.2, 80.8, 79.7, 77.8, 76.0, 75.6, 75.1, 74.3, 73.5, 70.2, 67.9, 67.8, 64.9, 29.7, 28.1, 26.3, 17.4; FTIR (neat) $\mathrm{cm}^{-1} 3063,3031,2984,2929,1454,1366,1086$; HRMS (ESI) $\mathrm{m} / \mathrm{z}$ : Calcd for $\mathrm{C}_{46} \mathrm{H}_{54} \mathrm{O}_{10} \mathrm{Na}(\mathrm{M}+\mathrm{Na}) 789.3615$, found 789.3639 .

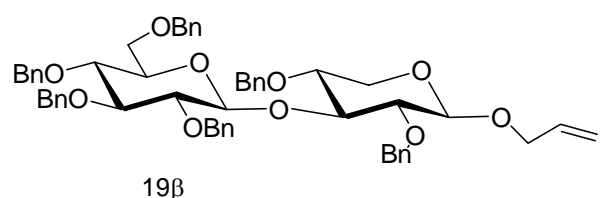

Disaccharide 19ß. Rf 0.50 (hexanes/ethyl acetate 3:1); ${ }^{1} \mathrm{H}$ NMR (400 MHz, $\left.\mathrm{CDCl}_{3}\right) \delta 7.40-7.15(\mathrm{~m}, 30 \mathrm{H}), 5.90(\mathrm{~m}, 1$ H), $5.29(\mathrm{~d}, \mathrm{~J}=17.2 \mathrm{~Hz}, 1 \mathrm{H}), 5.21(\mathrm{~d}, \mathrm{~J}=10.4 \mathrm{~Hz}, 1 \mathrm{H}), 5.05-4.81(\mathrm{~m}, 6 \mathrm{H}), 4.69-4.50(\mathrm{~m}, 6 \mathrm{H}), 4.39-4.31(\mathrm{~m}, 2 \mathrm{H})$, $4.11(\underline{A B X}, \mathrm{~J}=13.0,5.1 \mathrm{~Hz}, 1 \mathrm{H}), 3.94(\mathrm{ABX}, \mathrm{J}=13.0,7.0 \mathrm{~Hz}), 3.78-3.38(\mathrm{~m}, 11 \mathrm{H}) ;{ }^{13} \mathrm{C}$ NMR $\left(101 \mathrm{MHz}, \mathrm{CDCl}_{3}\right) \delta$ 139.1, 139.0, 138. 9, 138.8, 138.5, 138.2, 133.9, 128.6, 128.6, 128.5, 128.4, 128.2, 128.1,128.1, 128.0, 127.9, 127.8, 127.7, 127.6, 127.5, 118.6, 102.7, 95.2, 85.1, 83.5, 81.3, 78.4, 77.8, 76.0, 75.3, 75.2, 75.0, 73.6, 73.6, 60.3; FTIR (neat) $\mathrm{cm}^{-1} 3031,2867,1496,1453,1092$; HRMS (ESI) m/z: Calcd for $\mathrm{C}_{56} \mathrm{H}_{60} \mathrm{O}_{10} \mathrm{Na}(\mathrm{M}+\mathrm{Na})$ 915.4084, found 915.4053.

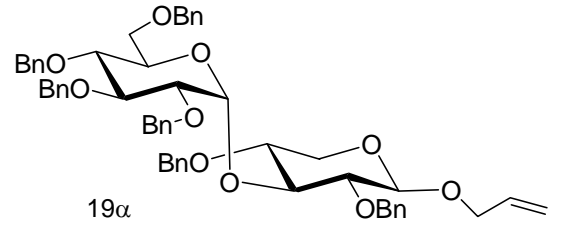

Disaccharide 19 $\alpha$. Rf 0.43 (hexanes/ethyl acetate 3:1); ${ }^{1} \mathrm{H}$ NMR $\left(400 \mathrm{MHz}, \mathrm{CDCl}_{3}\right) \delta$ 7.30-7.10 (m, $\left.30 \mathrm{H}\right), 5.91(\mathrm{~m}, 1$ $\mathrm{H}), 5.70(\mathrm{~d}, \mathrm{~J}=3.6 \mathrm{~Hz}, 1 \mathrm{H}), 5.34(\mathrm{dq}, \mathrm{J}=17.3,1.4 \mathrm{~Hz}, 1 \mathrm{H}), 5.21(\mathrm{dq}, \mathrm{J}=10.4,1.0 \mathrm{~Hz}, 1 \mathrm{H}), 4.97(\underline{\mathrm{AB}}, \mathrm{J}=10.9 \mathrm{~Hz}, 1$ H), 4.85-4.80 (m, $2 \mathrm{H}), 4.77(\underline{\mathrm{AB}}, \mathrm{J}=11.1 \mathrm{~Hz}, 1 \mathrm{H}), 4.63(\underline{\mathrm{AB}}, \mathrm{J}=12.0 \mathrm{~Hz}, 1 \mathrm{H}), 4.60-4.50(\mathrm{~m}, 6 \mathrm{H}), 4.39(\mathrm{~A} \underline{\mathrm{B}}, \mathrm{J}=$ $11.1 \mathrm{~Hz}, 1 \mathrm{H}), 4.30-4.21(\mathrm{~m}, 3 \mathrm{H}), 4.15(\mathrm{~m}, 1 \mathrm{H}), 4.02-3.92(\mathrm{~m}, 2 \mathrm{H}), 3.75(\mathrm{~m}, 1 \mathrm{H}), 3.68-3.61(\mathrm{~m}, 2 \mathrm{H}), 3.58-3.51(\mathrm{~m}$,

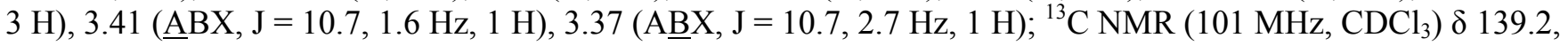
139.1, 138.4, 138.4, 138.3, 138.0, 134.1, 128.8, 128.6, 128.5, 128.5, 128.4, 128.3, 128.1, 128.0, 127.9, 127.8, 127.7, 127.7, 127.7, 127.6, 127.5, 127.1, 118.1, 97.0, 95.3, 82.3, 79.9, 79.6, 78.2, 78.1, 75.7, 74.9, 73.5, 73.0, 72.6, 72.3, 69.9, 68.3, 59.5; FTIR (neat) $\mathrm{cm}^{-1} 3062,3030,2869,1453,1362,1074$; HRMS (ESI) $\mathrm{m} / \mathrm{z}$ : Calcd for $\mathrm{C}_{56} \mathrm{H}_{60} \mathrm{O}_{10} \mathrm{Na}(\mathrm{M}+\mathrm{Na})$ 915.4084, found 915.4119 .

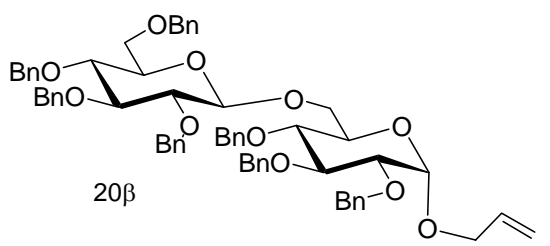

Disaccharide 20 $\beta$. Rf 0.23 (benzene/ethyl acetate 19:1); ${ }^{1} \mathrm{H}$ NMR $\left(400 \mathrm{MHz}, \mathrm{CDCl}_{3}\right) \delta 7.40-7.15(\mathrm{~m}, 35 \mathrm{H}), 5.87(\mathrm{~m}$, $1 \mathrm{H}), 5.25$ (dq, J = 17.2, $1.4 \mathrm{~Hz}, 1 \mathrm{H}), 5.17(\mathrm{dq}, \mathrm{J}=10.3,1.1 \mathrm{~Hz}, 1 \mathrm{H}), 4.98(\underline{\mathrm{AB}}, \mathrm{J}=10.9 \mathrm{~Hz}, 2 \mathrm{H}), 4.90(\underline{\mathrm{AB}}, \mathrm{J}=10.9$ $\mathrm{Hz}, 1 \mathrm{H}), 4.83-4.73(\mathrm{~m}, 6 \mathrm{H}), 4.70(\underline{\mathrm{AB}}, \mathrm{J}=11.1 \mathrm{~Hz}, 1 \mathrm{H}), 4.64(\mathrm{~A} \underline{\mathrm{B}}, \mathrm{J}=12.1 \mathrm{~Hz}, 1 \mathrm{H}), 4.59(\underline{\mathrm{AB}}, \mathrm{J}=12.2 \mathrm{~Hz}, 1 \mathrm{H})$, 4.57-4.48 (m, $3 \mathrm{H}), 4.35(\mathrm{~d}, \mathrm{~J}=7.8 \mathrm{~Hz}, 1 \mathrm{H}), 4.18(\mathrm{dd}, \mathrm{J}=10.8,1.8 \mathrm{~Hz}, 1 \mathrm{H}), 4.13\left(\underline{A B M X}_{2}, \mathrm{~J}=13.0,5.0,1.3 \mathrm{~Hz}, 1\right.$ $\mathrm{H}), 4.02(\mathrm{t}, \mathrm{J}=9.3 \mathrm{~Hz}, 1 \mathrm{H}), 3.97\left(\mathrm{ABMX}_{2}, \mathrm{~J}=13.0,5.2,1.3 \mathrm{~Hz}, 1 \mathrm{H}\right), 3.87(\mathrm{~m}, 1 \mathrm{H}), 3.74-3.48(\mathrm{~m}, 8 \mathrm{H}), 3.44(\mathrm{~m}, 1$ $\mathrm{H}) ;{ }^{13} \mathrm{C}$ NMR $\left(101 \mathrm{MHz}, \mathrm{CDCl}_{3}\right) \delta 139.1,138.7,138.5,138.5,138.4,138.3,138.2,133.8,128.6,128.6,128.5,128.5$, 
$128.3,128.2,128.1,128.1,128.0,128.0,127.9,127.8,127.8,127.8,127.7,118.5,103.9,95.6,85.0,82.2,82.1,79.9$, 78.1, 75.9, 75.9, 75.2, 75.1, 73.6, 73.4, 70.2, 69.2, 68.6, 68.2; FTIR (neat) $\mathrm{cm}^{-1} 3031,2910,1497,1454,1358,1070$; HRMS (ESI) $\mathrm{m} / z$ : Calcd for $\mathrm{C}_{64} \mathrm{H}_{68} \mathrm{O}_{11} \mathrm{Na}(\mathrm{M}+\mathrm{Na}) 1035.4659$, found 1035.4690 .

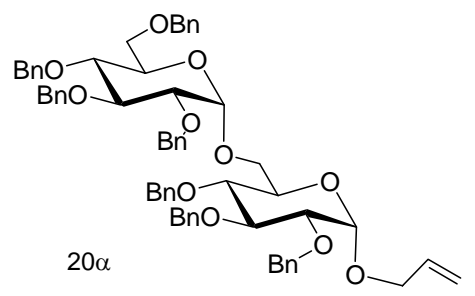

Disaccharide 20 $\alpha$. Rf 0.27 (methylenechloride/diethyl ether 60:1); ${ }^{1} \mathrm{H}$ NMR $\left(400 \mathrm{MHz}, \mathrm{CDCl}_{3}\right) \delta$ 7.35-7.10 (m, 35 $\mathrm{H}), 5.91(\mathrm{~m}, 1 \mathrm{H}), 5.31(\mathrm{~d}, \mathrm{~J}=17.2 \mathrm{~Hz}, 1 \mathrm{H}), 5.17(\mathrm{~d}, \mathrm{~J}=10.2 \mathrm{~Hz}, 1 \mathrm{H}), 4.99-4.90(\mathrm{~m}, 4 \mathrm{H}), 4.84-4.75(\mathrm{~m}, 4 \mathrm{H}), 4.69-$ $4.61(\mathrm{~m}, 4 \mathrm{H}), 4.58(\underline{\mathrm{AB}}, \mathrm{J}=12.2 \mathrm{~Hz}, 1 \mathrm{H}), 4.57(\mathrm{AB}, \mathrm{J}=12.1 \mathrm{~Hz}, 1 \mathrm{H}), 4.46-4.39(\mathrm{~m}, 2 \mathrm{H}), 4.19-4.13(\mathrm{~m}, 1 \mathrm{H}), 4.03-$ $3.93(\mathrm{~m}, 3 \mathrm{H}), 3.88-3.77(\mathrm{~m}, 3 \mathrm{H}), 3.70(\mathrm{AB}, \mathrm{J}=11.3 \mathrm{~Hz}, 1 \mathrm{H}), 3.67-5.99(\mathrm{~m}, 3 \mathrm{H}), 3.58-3.51(\mathrm{~m}, 2 \mathrm{H}), 3.45(\mathrm{dd}, \mathrm{J}=$ 9.4, 3.6 Hz, 1 H); FTIR (neat) $\mathrm{cm}^{-1} 3063,3031,2921,1454,1360,1073$; HRMS (ESI) m/z: Calcd for $\mathrm{C}_{64} \mathrm{H}_{68} \mathrm{O}_{11} \mathrm{Na}$ $(\mathrm{M}+\mathrm{Na})$ 1035.4659, found 1035.4694 .

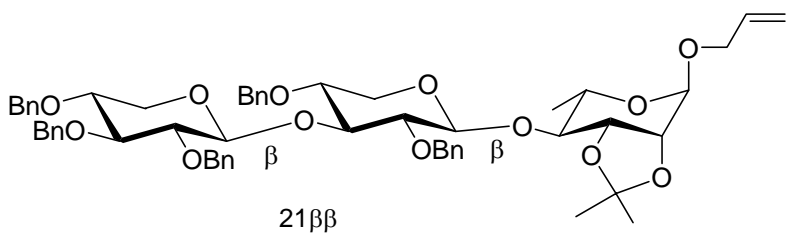

Trisaccharide 21 $\beta \beta$. Rf 0.33 (petroleum ether/ethyl acetate 5:1); ${ }^{1} \mathrm{H}$ NMR $\left(400 \mathrm{MHz}, \mathrm{CDCl}_{3}\right) \delta 7.40-7.15(\mathrm{~m}, 25 \mathrm{H})$, $5.90(\mathrm{~m}, 1 \mathrm{H}), 4.98(\underline{\mathrm{AB}}, \mathrm{J}=11.4 \mathrm{~Hz}, 1 \mathrm{H}), 4.93-4.86(\mathrm{AB}, \mathrm{J}=11.0 \mathrm{~Hz}, 2 \mathrm{H}), 4.91(\mathrm{~d}, \mathrm{~J}=7.6 \mathrm{~Hz}, 1 \mathrm{H}), 4.88(\mathrm{~d}, \mathrm{~J}=7.4$ $\mathrm{Hz}, 1 \mathrm{H}), 4.80(\mathrm{~d}, \mathrm{~J}=12.2 \mathrm{~Hz}, 2 \mathrm{H}), 4.73-4.68(\mathrm{~m}, 2 \mathrm{H}), 4.66(\mathrm{AB}, \mathrm{J}=12.1 \mathrm{~Hz}, 1 \mathrm{H}), 4.61(\mathrm{~A} \underline{\mathrm{B}}, \mathrm{J}=11.7 \mathrm{~Hz}, 1 \mathrm{H}), 4.51$ $(\mathrm{AB}, \mathrm{J}=10.1 \mathrm{~Hz}, 1 \mathrm{H}), 4.20-4.13(\mathrm{~m}, 2 \mathrm{H}), 4.10(\mathrm{AB}, \mathrm{J}=5.6 \mathrm{~Hz}, 1 \mathrm{H}), 4.02-3.87(\mathrm{~m}, 4 \mathrm{H}), 3.65-3.56(\mathrm{~m}, 3 \mathrm{H}), 3.54-$ $3.48(\mathrm{~m}, 2 \mathrm{H}), 3.36(\mathrm{t}, \mathrm{J}=7.6 \mathrm{~Hz}, 1 \mathrm{H}), 3.26(\mathrm{dd}, \mathrm{J}=8.7,7.6 \mathrm{~Hz}, 1 \mathrm{H}), 3.22(\mathrm{dd}, \mathrm{J}=11.5,10.0 \mathrm{~Hz}, 1 \mathrm{H}), 3.04(\mathrm{t}, \mathrm{J}=$ $10.4 \mathrm{~Hz}, 1 \mathrm{H}), 1.48(\mathrm{~s}, 3 \mathrm{H}), 1.32(\mathrm{~s}, 3 \mathrm{H}), 1.25(\mathrm{~d}, \mathrm{~J}=5.5 \mathrm{~Hz}, 3 \mathrm{H}) ;{ }^{13} \mathrm{C} \mathrm{NMR}\left(101 \mathrm{MHz}, \mathrm{CDCl}_{3}\right) \delta 138.9,138.9$, $138.7,138.5,138.4,133.8,128.8,128.7,128.6,128.5,128.4,128.2,128.2,128.2,128.1,127.9,127.8,127.8,127.7$, 118.0, 109.5, 103.4, 101.8, 96.3, 84.1, 82.8, 79.8, 78.4, 78.2, 76.2, 75.9, 75.5, 75.2, 74.8, 73.5, 73.3, 68.2, 64.4, 64.1, 63.9, 28.0, 26.5, 17.8; FTIR (neat) $\mathrm{cm}^{-1} 3030,2982,2929,1496,1452$, 1074; HRMS (ESI) $\mathrm{m} / \mathrm{z}$ : Calcd for $\mathrm{C}_{57} \mathrm{H}_{66} \mathrm{O}_{13} \mathrm{Na}_{2}$ $(\mathrm{M}+\mathrm{Na})$ 981.4401, found 981.4420 .

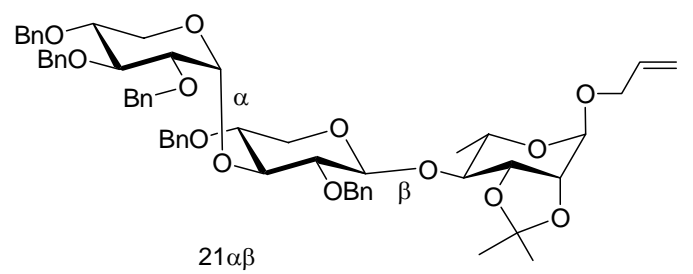

Trisaccharide $21 \alpha \beta$. Rf 0.27 (petroleum ether/diethyl ether 4:1); ${ }^{1} \mathrm{H}$ NMR $\left(400 \mathrm{MHz}, \mathrm{CDCl}_{3}\right) \delta 7.45-7.15(\mathrm{~m}, 25 \mathrm{H})$, $5.91(\mathrm{~m}, 1 \mathrm{H}), 5.56(\mathrm{~d}, \mathrm{~J}=3.6 \mathrm{~Hz}, 1 \mathrm{H}), 5.33(\mathrm{~d}, \mathrm{~J}=17.2 \mathrm{~Hz}, 1 \mathrm{H}), 5.21(\mathrm{~d}, \mathrm{~J}=10.4 \mathrm{~Hz}, 1 \mathrm{H}), 5.01(\mathrm{~s}, 1 \mathrm{H}), 4.97(\mathrm{~d}, \mathrm{~J}=$ $7.8 \mathrm{~Hz}, 1 \mathrm{H}), 4.92(\mathrm{~s}, 2 \mathrm{H}), 4.88(\underline{\mathrm{AB}}, \mathrm{J}=10.5 \mathrm{~Hz}, 1 \mathrm{H}), 4.72(\mathrm{AB}, \mathrm{J}=10.5 \mathrm{~Hz}, 1 \mathrm{H}), 4.66-4.55(\mathrm{M}, 4 \mathrm{H}), 4.52(\mathrm{~A} \underline{\mathrm{B}}, \mathrm{J}$ $=11.8 \mathrm{~Hz}, 1 \mathrm{H}), 4.22-4.17(\mathrm{~m}, 2 \mathrm{H}), 4.11(\mathrm{~d}, \mathrm{~J}=5.7 \mathrm{~Hz}, 1 \mathrm{H}), 4.02-3.89(\mathrm{~m}, 5 \mathrm{H}), 3.78(\mathrm{~m}, 1 \mathrm{H}), 3.69-3.62(\mathrm{~m}, 2 \mathrm{H})$, $3.51(\mathrm{~m}, 1 \mathrm{H}), 3.46-3.37(\mathrm{~m}, 3 \mathrm{H}), 3.2(\mathrm{~m}, 1 \mathrm{H}), 1.51(\mathrm{~s}, 3 \mathrm{H}), 1.35(\mathrm{~s}, 3 \mathrm{H}), 1.25(\mathrm{~d}, \mathrm{~J}=5.2 \mathrm{~Hz}, 3 \mathrm{H}) .{ }^{13} \mathrm{C}$ NMR $(101$ $\left.\mathrm{MHz}_{2} \mathrm{CDCl}_{3}\right) \delta 139.1,138.8,138.4,138.3,138.3,133.9,129.0,128.6,128.5,128.5,128.4,128.2,127.9,127.9,127.8$, $127.8,127.7,127.3,118.0,109.5,102.1,96.7,96.3,81.4,80.1,79.8,79.4,78.7 .78 .3,78.0,76.2,75.9,75.0,73.2,73.0$, 72.4, 68.2, 64.5, 63.2, 60.3, 29.9, 28.0, 26.6, 17.9; FTIR (neat) $\mathrm{cm}^{-1} 3031,2983,2908,1454,1371,1085$; HRMS (ESI) $\mathrm{m} / \mathrm{z}$ : Calcd for $\mathrm{C}_{57} \mathrm{H}_{66} \mathrm{O}_{13} \mathrm{Na}(\mathrm{M}+\mathrm{Na})$ 981.4401, found 981.4433 . 


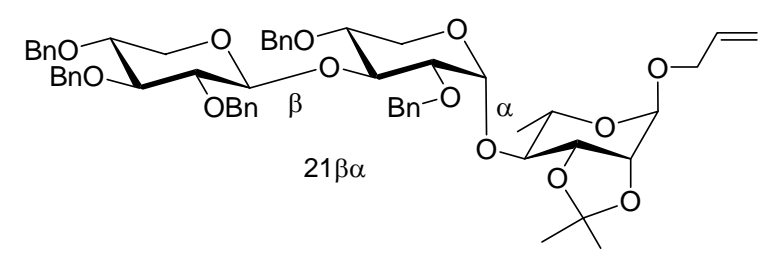

Trisaccharide 21 $\beta \alpha$. Rf 0.30 (petroleum ether/ethyl acetate 5:1); ${ }^{1} \mathrm{H}$ NMR (400 MHz, CDCl $) \delta$ 7.40-7.15 (m, $25 \mathrm{H}$ ), $5.89(\mathrm{~m}, 1 \mathrm{H}), 5.29(\mathrm{dq}, \mathrm{J}=17.2,1.54 \mathrm{~Hz}, 1 \mathrm{H}), 5.20(\mathrm{dq}, \mathrm{J}=10.4,1.1 \mathrm{~Hz}, 1 \mathrm{H}), 4.98(\mathrm{~s}, 1 \mathrm{H}), 4.95(\underline{\mathrm{AB}}, \mathrm{J}=11.4 \mathrm{~Hz}$, $1 \mathrm{H}), 4.91(\mathrm{~d}, \mathrm{~J}=7.7 \mathrm{~Hz}, 1 \mathrm{H}), 4.90(\mathrm{~s}, 2 \mathrm{H}), 4.86-4.80(\mathrm{~m}, 3 \mathrm{H}), 4.71(\underline{\mathrm{AB}}, \mathrm{J}=11.7 \mathrm{~Hz}, 1 \mathrm{H}), 4.68-4.58(\mathrm{~m}, 3 \mathrm{H}), 4.41$ $(\mathrm{AB}, \mathrm{J}=11.0 \mathrm{~Hz}, 1 \mathrm{H}), 4.22(\mathrm{t}, \mathrm{J}=9.3 \mathrm{~Hz}, 1 \mathrm{H}), 4.17-4.09(\mathrm{~m}, 3 \mathrm{H}), 3.99-3.91(\mathrm{~m}, 3 \mathrm{H}), 3.74(\mathrm{~m}, 1 \mathrm{H}), 3.63(\mathrm{~m}, 1 \mathrm{H})$, 3.59-3.52 (m, 2 H), 3.49-3.32 (m, $3 \mathrm{H}), 3.30$ (dd, J = 10.0, $7.0 \mathrm{~Hz}, 1 \mathrm{H}), 3.14$ (t, J = 11.3 Hz, $1 \mathrm{H}), 1.46(\mathrm{~s}, 3 \mathrm{H}), 1.31$ $(\mathrm{s}, 3 \mathrm{H}), 1.24(\mathrm{~d}, \mathrm{~J}=6.3 \mathrm{~Hz}, 3 \mathrm{H}) ;{ }^{13} \mathrm{C} \mathrm{NMR}\left(101 \mathrm{MHz}, \mathrm{CDCl}_{3}\right) \delta 138.9,138.8,138.4,137.9,133.8,128.7,128.5$, 128.5, 128.4, 128.2, 128.1, 128.0, 127.8, 127.7, 118.0, 109.1, 103.1, 97.6, 96.1, 84.2, 83.0, 81.4, 80.7, 78.5, 76.2, 76.2, 75.8, 75.3, 74.3, 3.3, 73.4, 68.0, 65.0,63.9, 60.7, 28.0, 26.6, 17.8; FTIR (neat) $\mathrm{cm}^{-1} 3033,2985,2906,1497,1368,1267$, 1078; HRMS (ESI) m/z: Calcd for $\mathrm{C}_{57} \mathrm{H}_{66} \mathrm{O}_{13} \mathrm{Na}(\mathrm{M}+\mathrm{Na})$ 981.4401, found 981.4430.

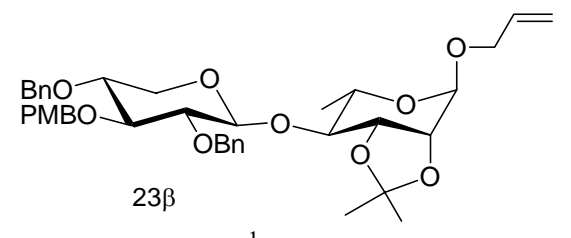

Disaccharide 22 $\beta$. Rf 0.25 (benzene/ethyl acetate 19:1); ${ }^{1} \mathrm{H}$ NMR (400 MHz, $\left.\mathrm{CDCl}_{3}\right) \delta$ 7.40-7.20 (m, $\left.12 \mathrm{H}\right), 6.85(\mathrm{~d}, \mathrm{~J}$ $=8.5 \mathrm{~Hz}, 2 \mathrm{H}), 5.91(\mathrm{~m}, 1 \mathrm{H}), 5.34(\mathrm{dq}, \mathrm{J}=17.2,1.6 \mathrm{~Hz}, 1 \mathrm{H}), 5.22(\mathrm{dq}, \mathrm{J}=10.4,1.5 \mathrm{~Hz}, 1 \mathrm{H}), 5.01(\mathrm{~s}, 1 \mathrm{H}), 4.91(\mathrm{~d}, \mathrm{~J}$ $=7.6 \mathrm{~Hz}, 1 \mathrm{H}), 4.89(\underline{\mathrm{AB}}, \mathrm{J}=11.1 \mathrm{~Hz}, 1 \mathrm{H}), 4.80-4.75(\mathrm{AB}, \mathrm{J}=10.6 \mathrm{~Hz}, 2 \mathrm{H}), 4.71(\underline{\mathrm{AB}}, \mathrm{J}=11.7 \mathrm{~Hz}, 1 \mathrm{H}), 4.69(\mathrm{~A} \underline{\mathrm{B}}$, $\mathrm{J}=11.1 \mathrm{~Hz}, 1 \mathrm{H}), 4.62(\mathrm{~A} \underline{\mathrm{B}}, \mathrm{J}=11.7 \mathrm{~Hz}, 1 \mathrm{H}), 4.24-4.15(\mathrm{~m}, 2 \mathrm{H}), 4.12(\mathrm{AB}, \mathrm{J}=5.7 \mathrm{~Hz}, 1 \mathrm{H}), 4.01\left(\mathrm{~A} \underline{B} M X_{2}, \mathrm{~J}=\right.$ 12.7, 6.2, $1.3 \mathrm{~Hz}, 1 \mathrm{H}), 3.93(\mathrm{~m}, 1 \mathrm{H}), 3.80(\mathrm{~s}, 3 \mathrm{H}), 3.67-3.57(\mathrm{~m}, 4 \mathrm{H}), 3.29(\mathrm{~m}, 1 \mathrm{H}), 3.20(\mathrm{~m}, 1 \mathrm{H}), 1.51(\mathrm{~s}, 3 \mathrm{H})$, $1.34(\mathrm{~s}, 1 \mathrm{H}), 1.26(\mathrm{~d}, \mathrm{~J}=5.7 \mathrm{~Hz}, 3 \mathrm{H}) ;{ }^{13} \mathrm{C} \mathrm{NMR}\left(101 \mathrm{MHz}, \mathrm{CDCl}_{3}\right) \delta 159.3,138.9,138.5,138.9,131.1,129.8,128.6$, 128.5, 128.3,128.0, 127.8, 118.0, 113.9, 109.5, 102.1, 96.3, 83.8, 82.2, 78.4, 78,2, 78.2, 76.2, 75.5, 75.0, 73.4, 68.2,64.5,64.0, 55.5, 28.0, 26.6, 17.8; FTIR (neat) $\mathrm{cm}^{-1}$ 2984, 2934, 1612, 1514, 1247, 1085; HRMS (ESI) m/z: Calcd for $\mathrm{C}_{39} \mathrm{H}_{48} \mathrm{O}_{10} \mathrm{Na}(\mathrm{M}+\mathrm{Na}) 699.3145$, found 699.3168 .

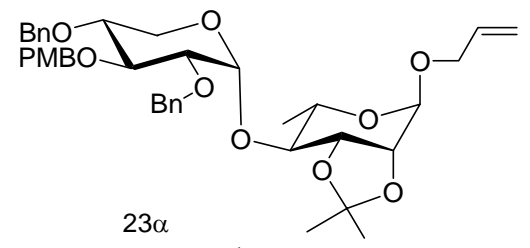

Disaccharide 22 $\alpha$. Rf 0.17 (benzene/ethyl acetate 19:1); ${ }^{1} \mathrm{H}$ NMR (400 MHz, $\left.\mathrm{CDCl}_{3}\right) \delta$ 7.40-7.25 (m, $\left.12 \mathrm{H}\right), 6.85(\mathrm{~d}, \mathrm{~J}$ $=8.5 \mathrm{~Hz}, 2 \mathrm{H}), 5.90(\mathrm{~m}, 1 \mathrm{H}), 5.31(\mathrm{dq}, \mathrm{J}=16.8,1.5 \mathrm{~Hz}, 1 \mathrm{H}), 5.22(\mathrm{dq}, \mathrm{J}=10.4,0.86 \mathrm{~Hz}, 1 \mathrm{H}), 5.01(\mathrm{~s}, 1 \mathrm{H}), 4.88(\mathrm{~d}$, $\mathrm{J}=3.6 \mathrm{~Hz}, 1 \mathrm{H}), 4.82(\mathrm{~s}, 2 \mathrm{H}), 4.81(\underline{\mathrm{AB}}, \mathrm{J}=11.5 \mathrm{~Hz}, 1 \mathrm{H}), 4.77-4.66(\mathrm{AB}, \mathrm{J}=11.7 \mathrm{~Hz}, 2 \mathrm{H}), 4.65(\mathrm{AB}, \mathrm{J}=11.7 \mathrm{~Hz}$, $1 \mathrm{H}), 4.20-4.12(\mathrm{~m}, 3 \mathrm{H}), 4.00-3.78(\mathrm{~m}, 7 \mathrm{H}), 3.61-3.51(\mathrm{~m}, 2 \mathrm{H}), 3.46(\mathrm{dd}, \mathrm{J}=9.7,3.7 \mathrm{~Hz}, 1 \mathrm{H}), 3.37$ (dd, J = 10.0, 7.5 $\mathrm{Hz}, 1 \mathrm{H}), 1.45$ (s, $3 \mathrm{H}), 1.30$ (s, $3 \mathrm{H}), 1.28$ (d, J = 6.3 Hz, $3 \mathrm{H}) ;{ }^{13} \mathrm{C} \mathrm{NMR}\left(101 \mathrm{MHz}, \mathrm{CDCl}_{3}\right) \delta 159.3,138.6,138.3$, 133.8, 131.2, 129.8, 128.6, 128.4, 128.1, 128.0, 118.0, 114.0, 109.2, 98.3, 96.2, 81.5, 80.6, 78.0, 78.7, 76.4, 75.6, 74.5, 73.6, 68.0, 65.3, 60.7, 55.5, 28.0, 26.7, 17.7; FTIR (neat) $\mathrm{cm}^{-1} 2905,1513,1457,1246,1086$; HRMS (ESI) m/z: Calcd for $\mathrm{C}_{39} \mathrm{H}_{48} \mathrm{O}_{10} \mathrm{Na}(\mathrm{M}+\mathrm{Na}) 699.3145$, found 699.3175 .

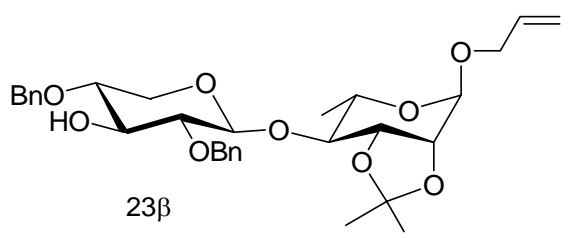

Disaccharide 23ß. Rf 0.23 (petroleum ether/ethyl acetate 5:1); ${ }^{1} \mathrm{H} \mathrm{NMR}\left(400 \mathrm{MHz}, \mathrm{CDCl}_{3}\right) \delta$ 7.40-7.20 (m, $\left.10 \mathrm{H}\right)$, $5.91(\mathrm{~m}, 1 \mathrm{H}), 5.32(\mathrm{dq}, \mathrm{J}=17.2,1.6 \mathrm{~Hz}, 1 \mathrm{H}), 5.22(\mathrm{ddt}, \mathrm{J}=10.4,1.6,1.2 \mathrm{~Hz}, 1 \mathrm{H}), 5.02(\mathrm{~s}, 1 \mathrm{H}), 4.92(\underline{\mathrm{AB}}, \mathrm{J}=11.5$ $\mathrm{Hz}, 1 \mathrm{H}), 4.90(\mathrm{~d}, \mathrm{~J}=7.5 \mathrm{~Hz}, 1 \mathrm{H}), 4.74(\underline{\mathrm{AB}}, \mathrm{J}=11.9 \mathrm{~Hz}, 1 \mathrm{H}), 4.65(\mathrm{~A} \underline{\mathrm{B}}, \mathrm{J}=11.5 \mathrm{~Hz}, 1 \mathrm{H}), 4.63(\mathrm{AB}, \mathrm{J}=11.9 \mathrm{~Hz}, 1$ $\mathrm{H}), 4.23-4.15(\mathrm{~m}, 2 \mathrm{H}), 4.13-4.10(\mathrm{~m}, 1 \mathrm{H}), 4.00\left(\mathrm{ABMX}_{2}, \mathrm{~J}=12.8,6.2,1.3 \mathrm{~Hz}, 1 \mathrm{H}\right), 3.94(\mathrm{dd}, \mathrm{J}=11.6,5.3 \mathrm{~Hz}, 1 \mathrm{H})$, 3.73-3.60 (m, 3 H), $3.52(\mathrm{~m}, 1 \mathrm{H}), 3.21(\mathrm{dd}, \mathrm{J}=11.6,9.7 \mathrm{~Hz}, 1 \mathrm{H}), 3.17(\mathrm{dd}, \mathrm{J}=9.1,7.6 \mathrm{~Hz}, 1 \mathrm{H}), 2.56(\mathrm{~d}, \mathrm{~J}=2.1 \mathrm{~Hz}$, 
$1 \mathrm{H}), 1.50$ (s, $3 \mathrm{H}), 1.34$ (s, $3 \mathrm{H}), 1.27(\mathrm{~d}, \mathrm{~J}=5.7 \mathrm{~Hz}, 3 \mathrm{H}) ;{ }^{13} \mathrm{C}$ NMR $\left(101 \mathrm{MHz}, \mathrm{CDCl}_{3}\right) \delta 138.7,138.4,133.8,128.7$, 128.6, 128.3, 128.1, 128.0, 127.99, 127.98, 118.0, 109.5, 101.7, 96.3, 81.3, 78.4, 78.2, 76.9, 76.3, 75.8, 74.5, 73.1, 68.2, 64.4, 64.0, 28.0, 26.6, 17.8; FTIR (neat) $\mathrm{cm}^{-1} 3486,3064,3030,2984,2934,1497,1454,1372,1242,1221$, 1084, 1026; HRMS (ESI) m/z: Calcd for $\mathrm{C}_{31} \mathrm{H}_{40} \mathrm{O}_{9} \mathrm{Na}(\mathrm{M}+\mathrm{Na}) 579.2570$, found 579.2580. 


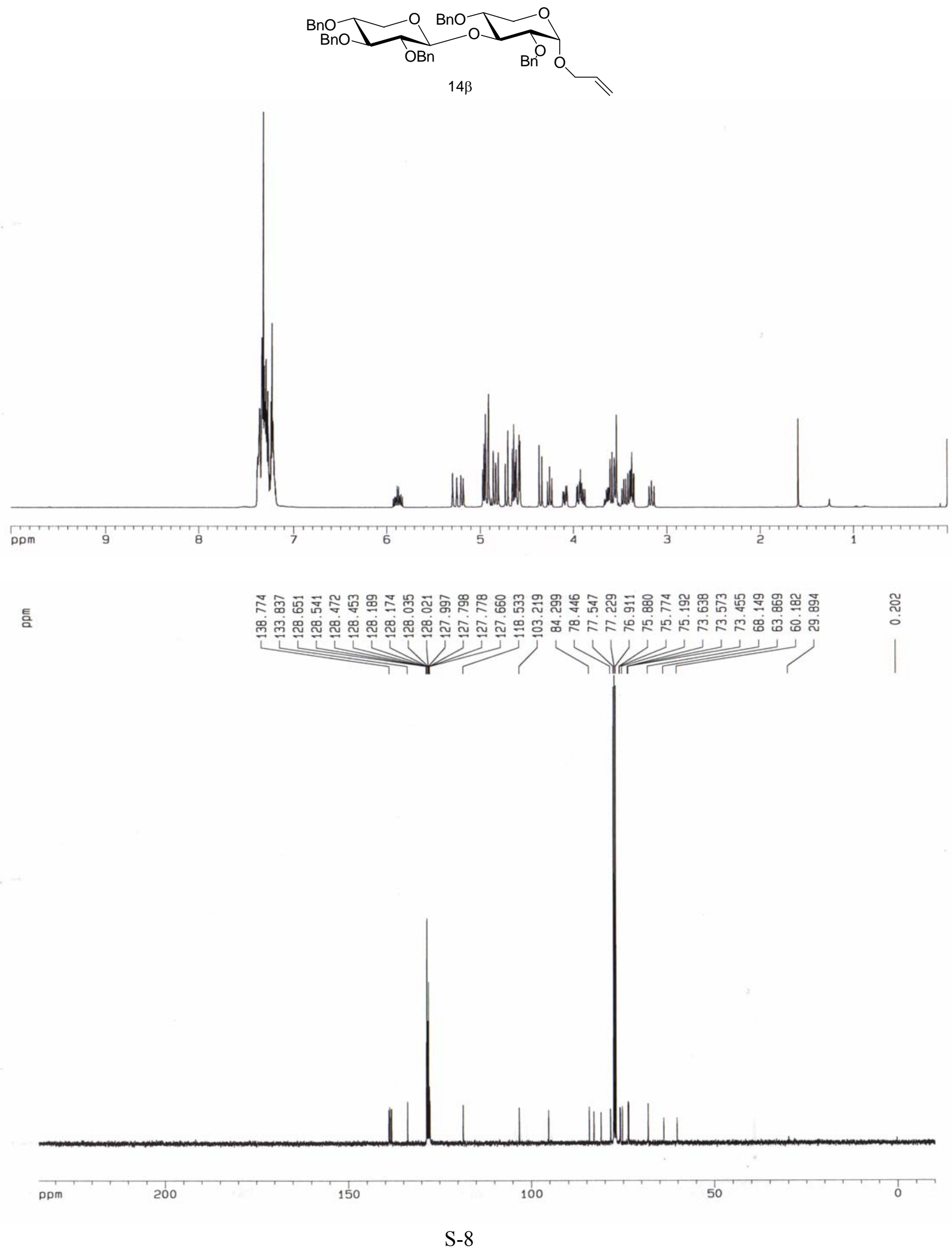



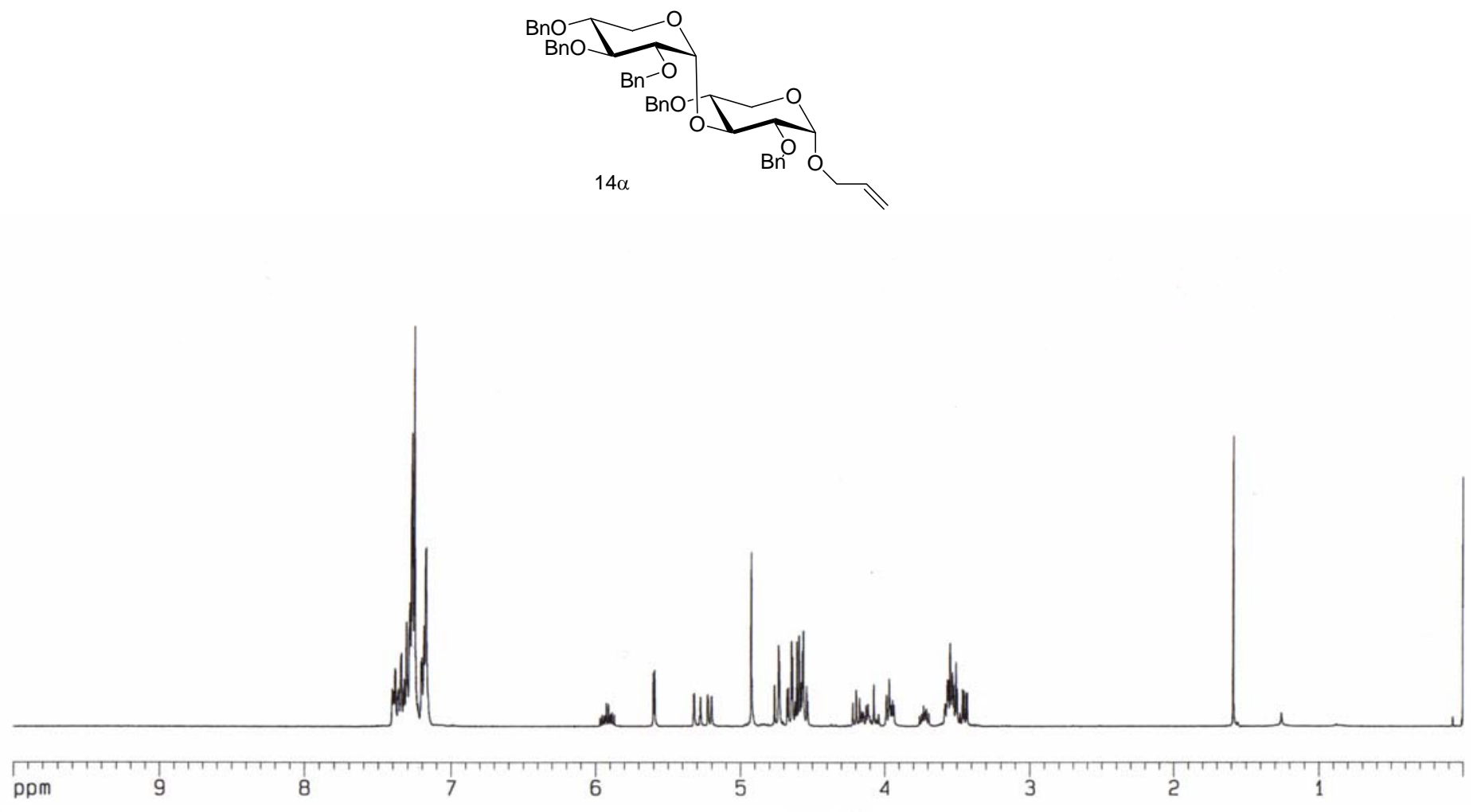

틈

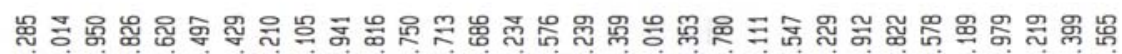

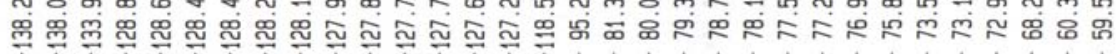

웡

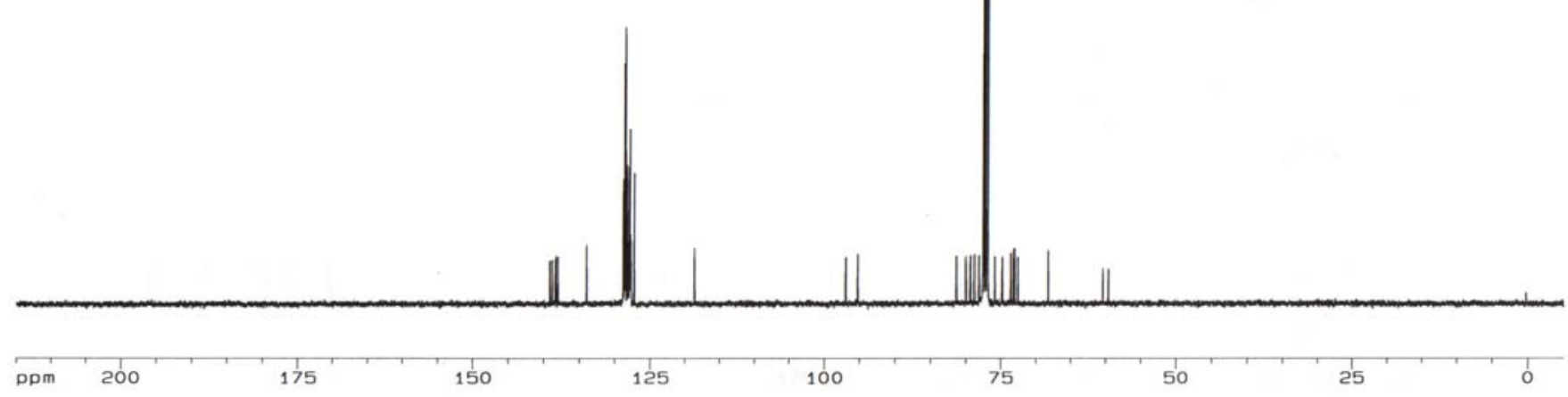

S-9 


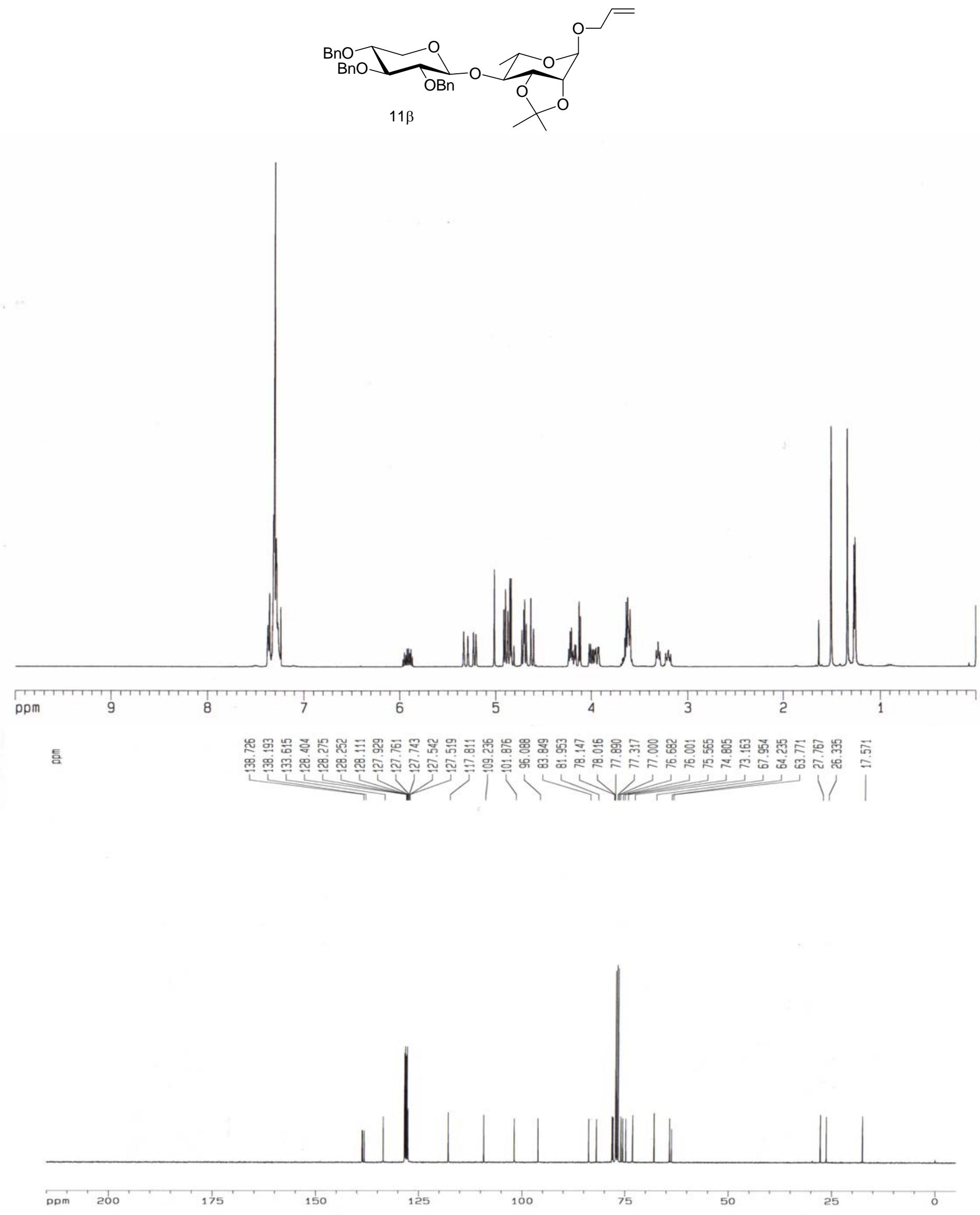

S-10 

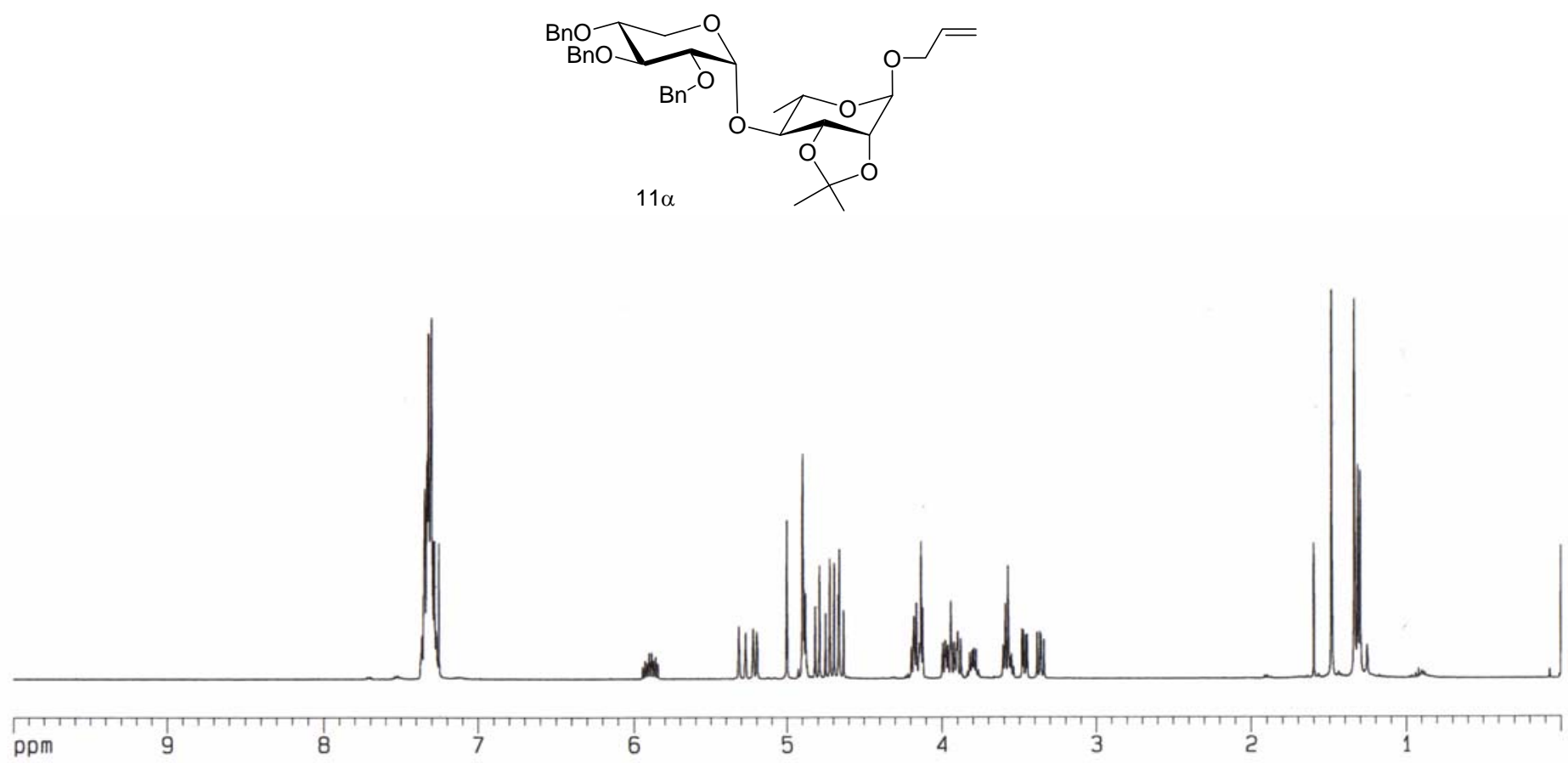

言

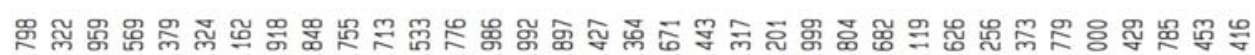

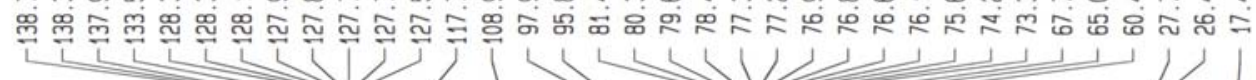

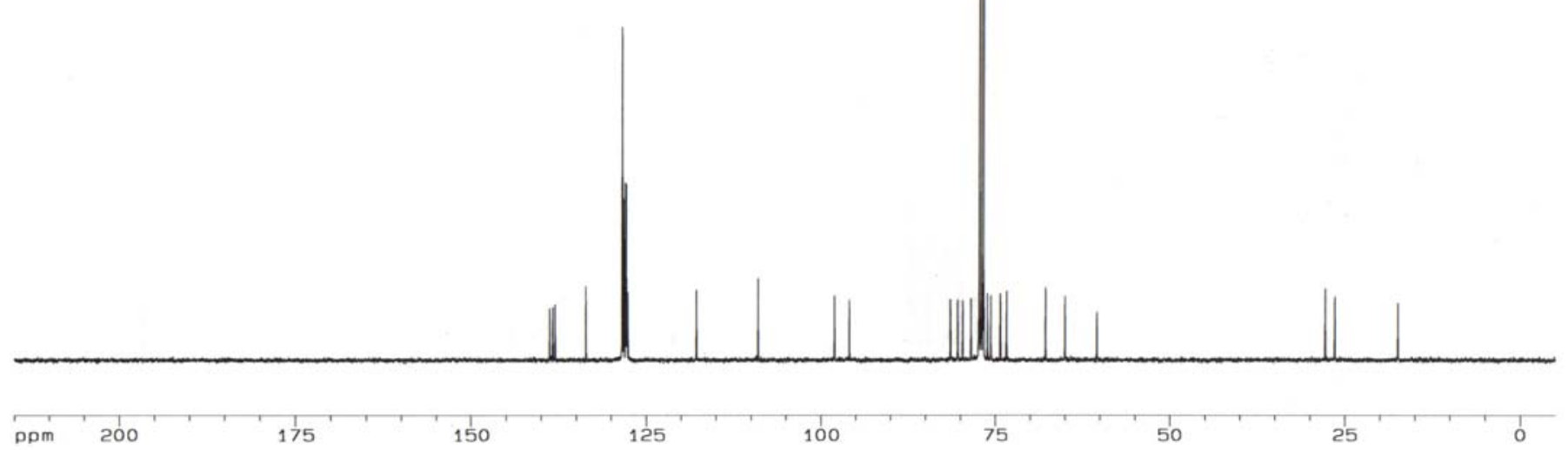




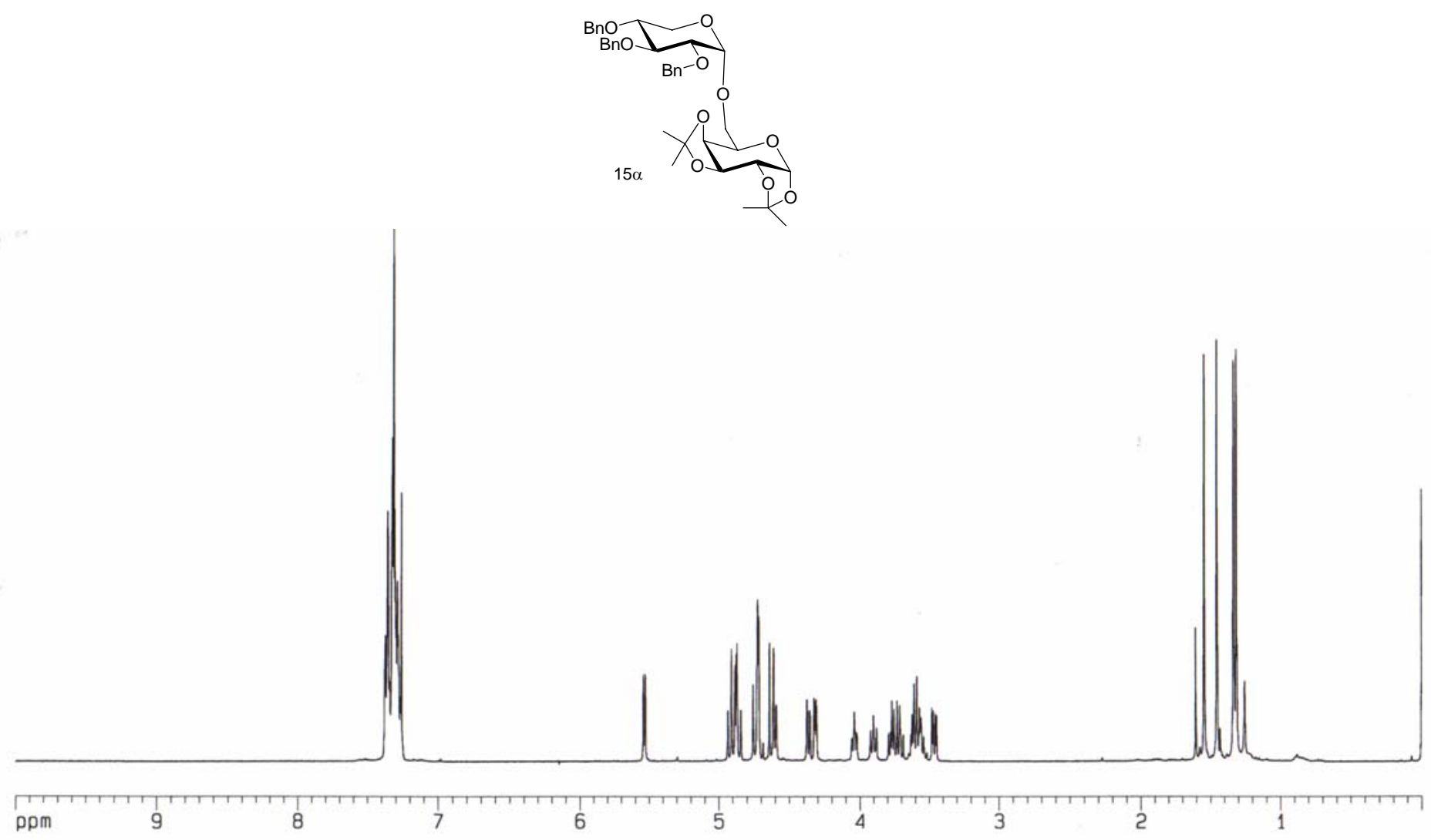

言

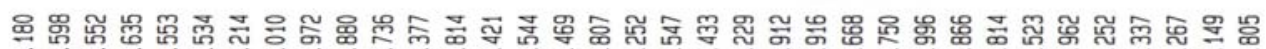

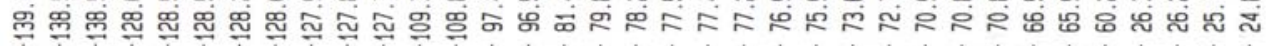
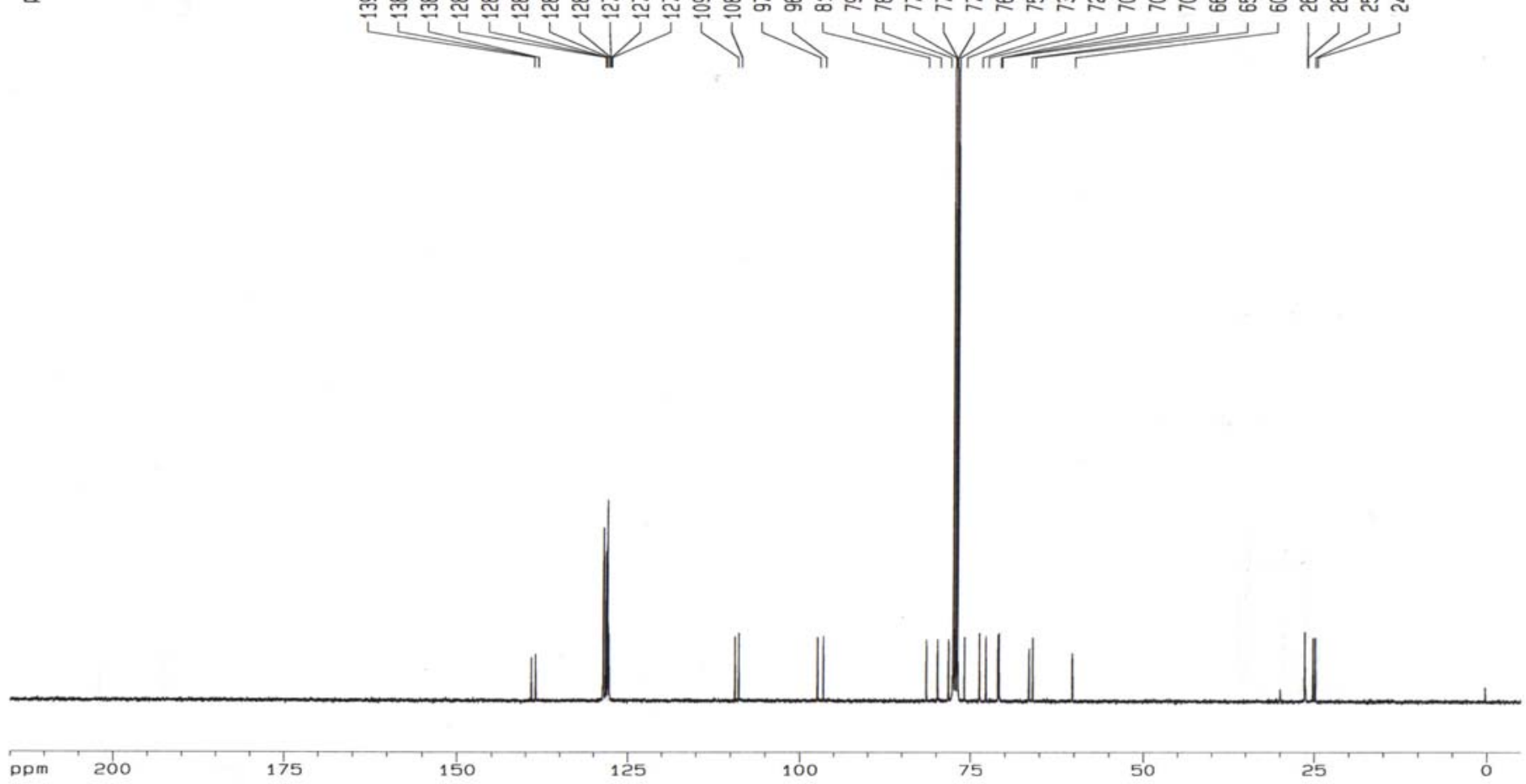


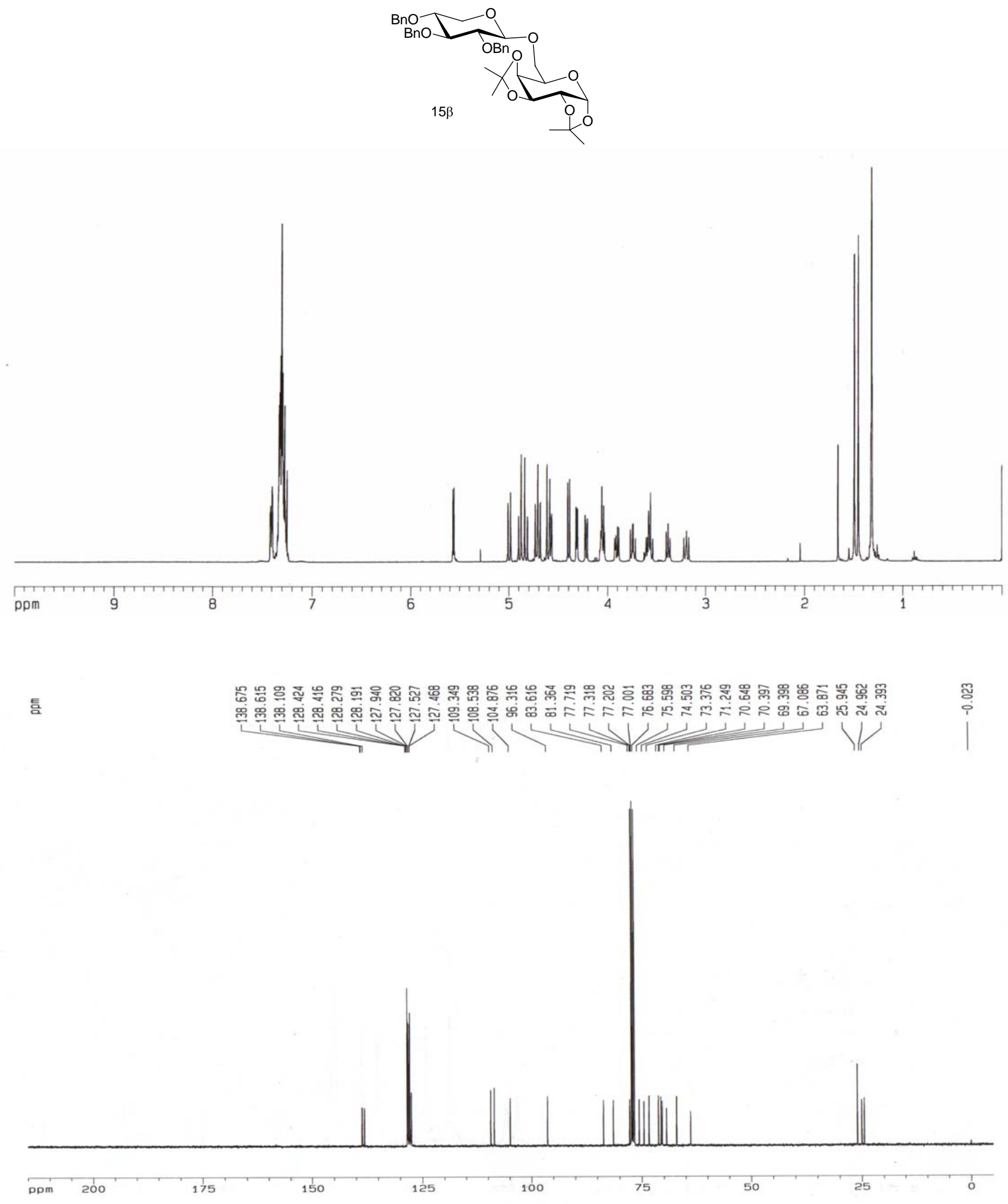



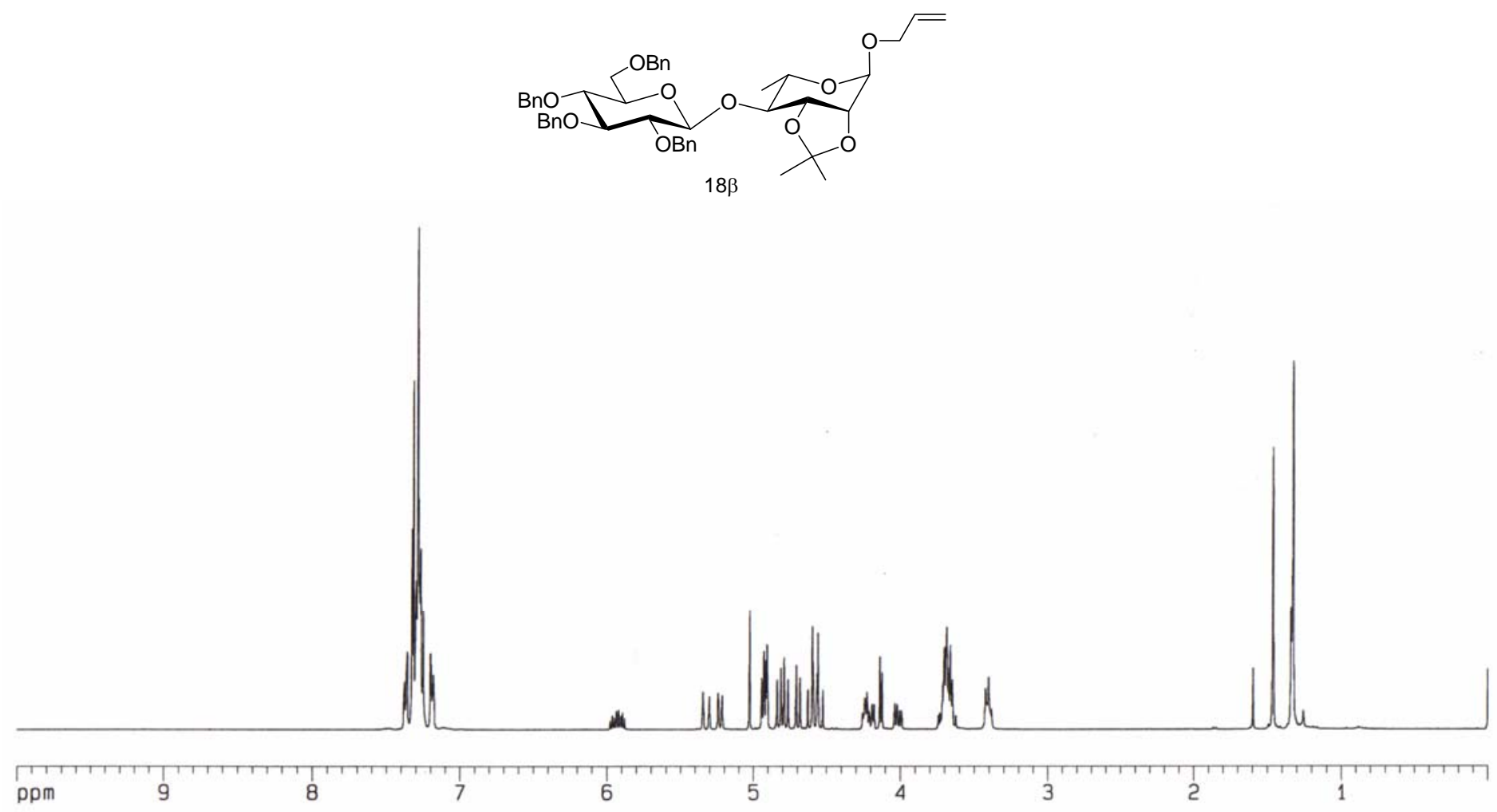

言

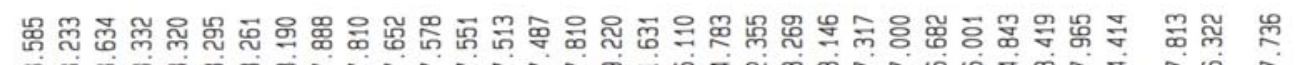

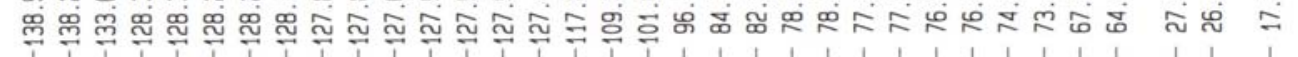
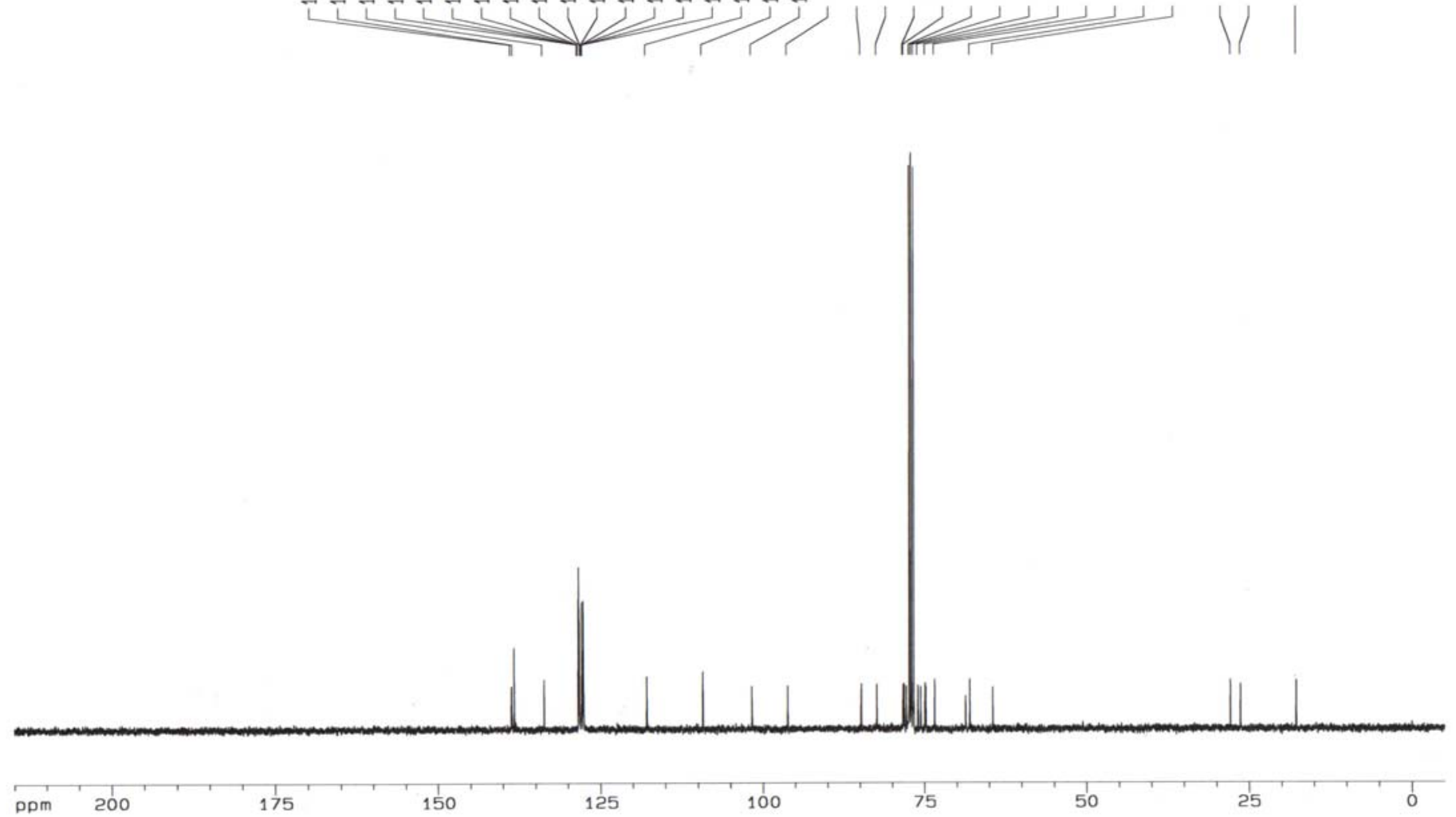

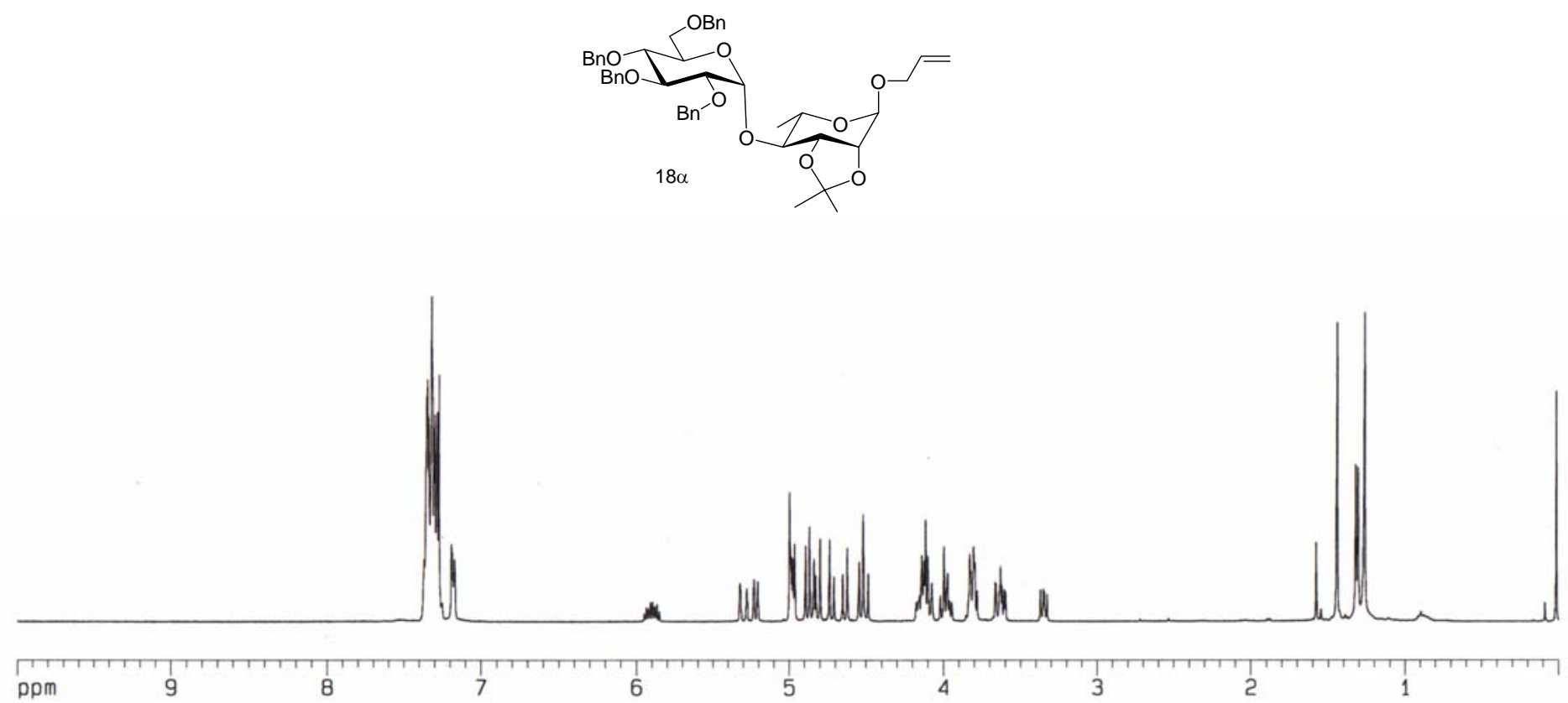

言

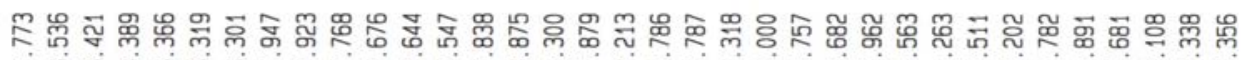

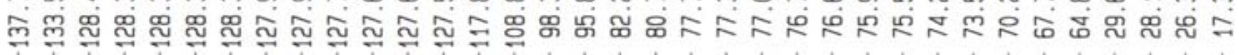
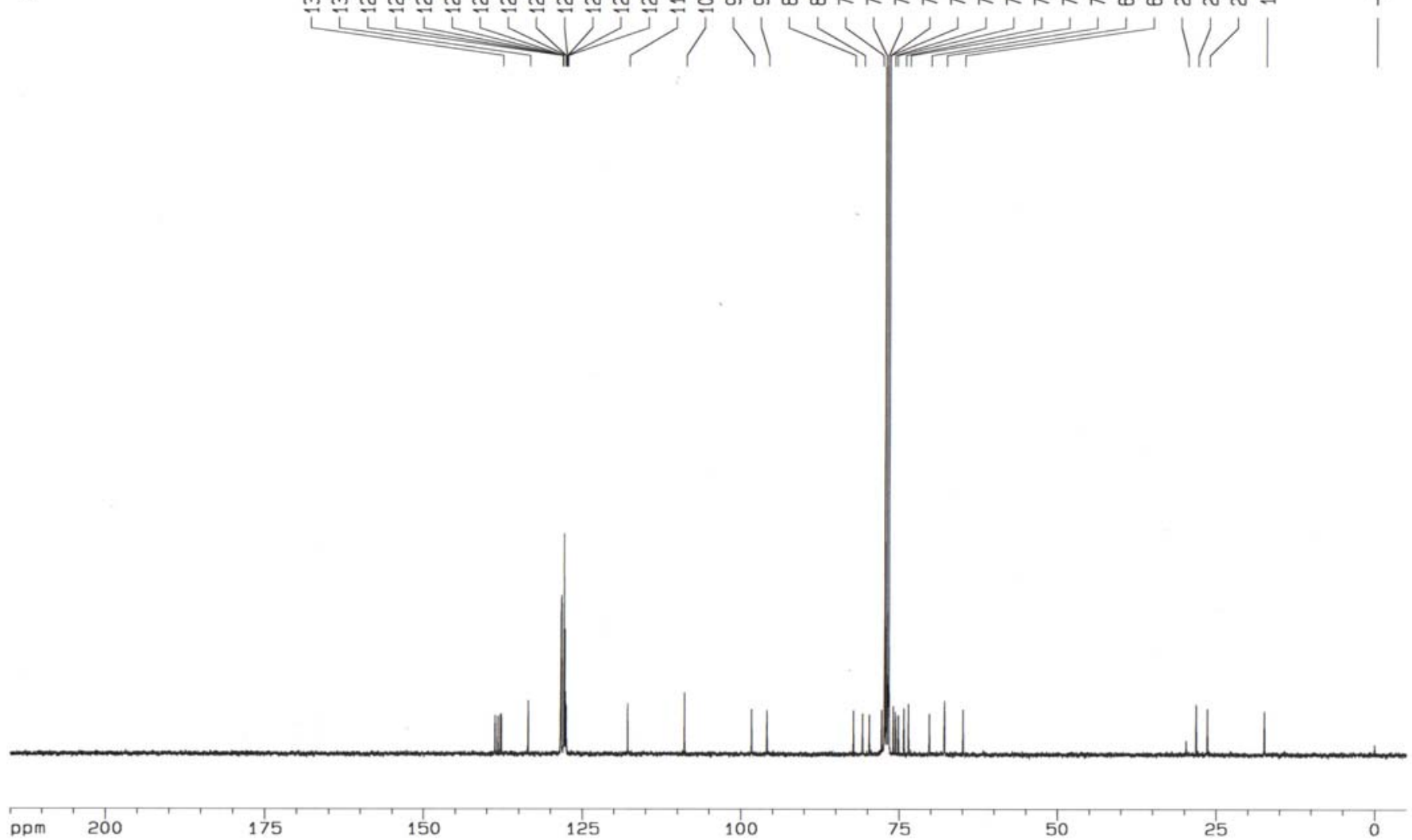


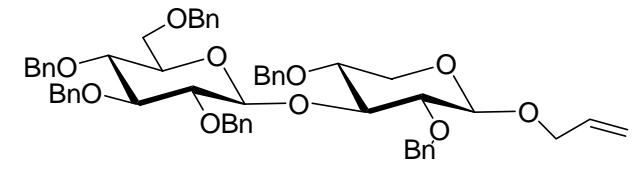

$19 \beta$
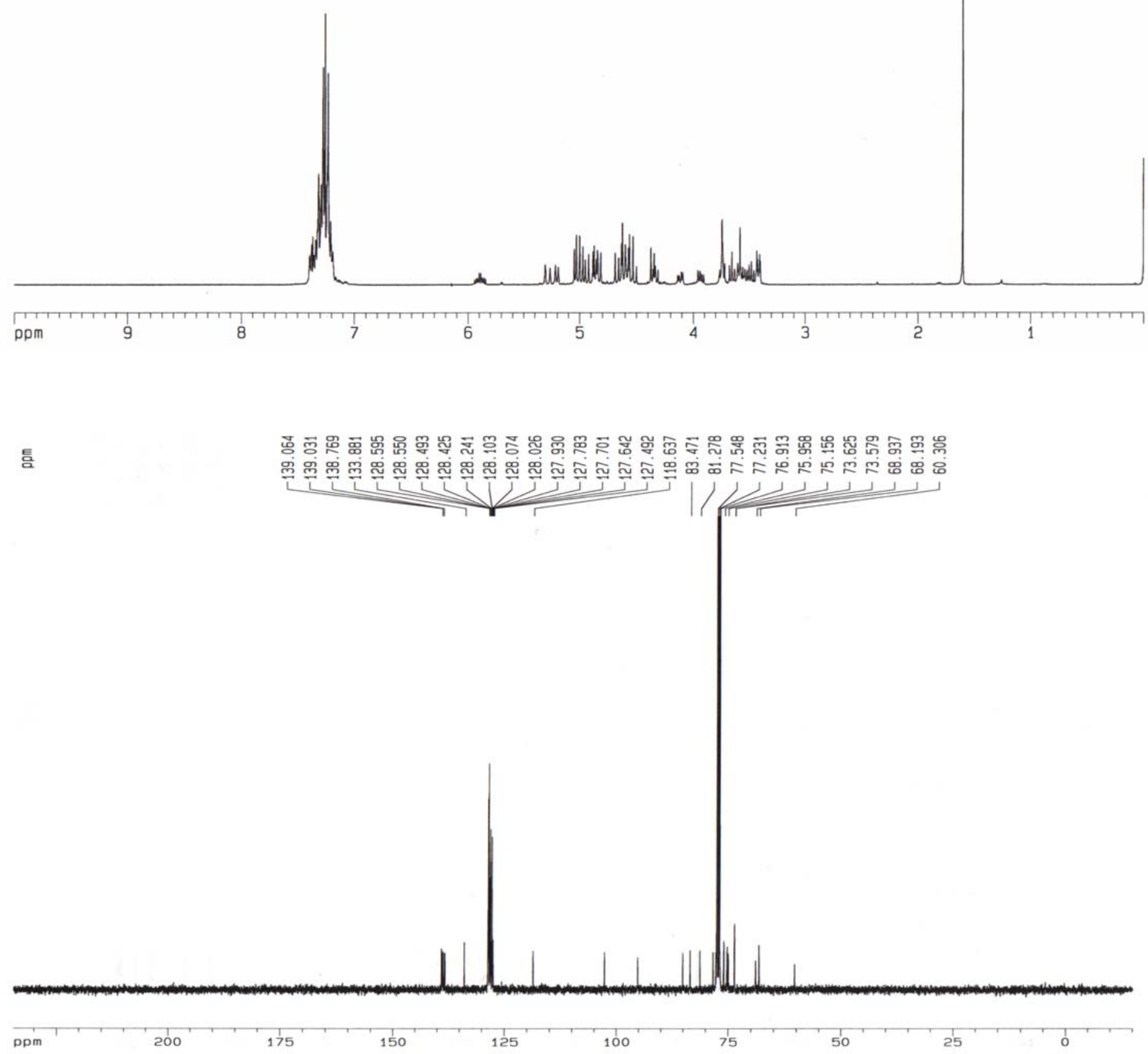


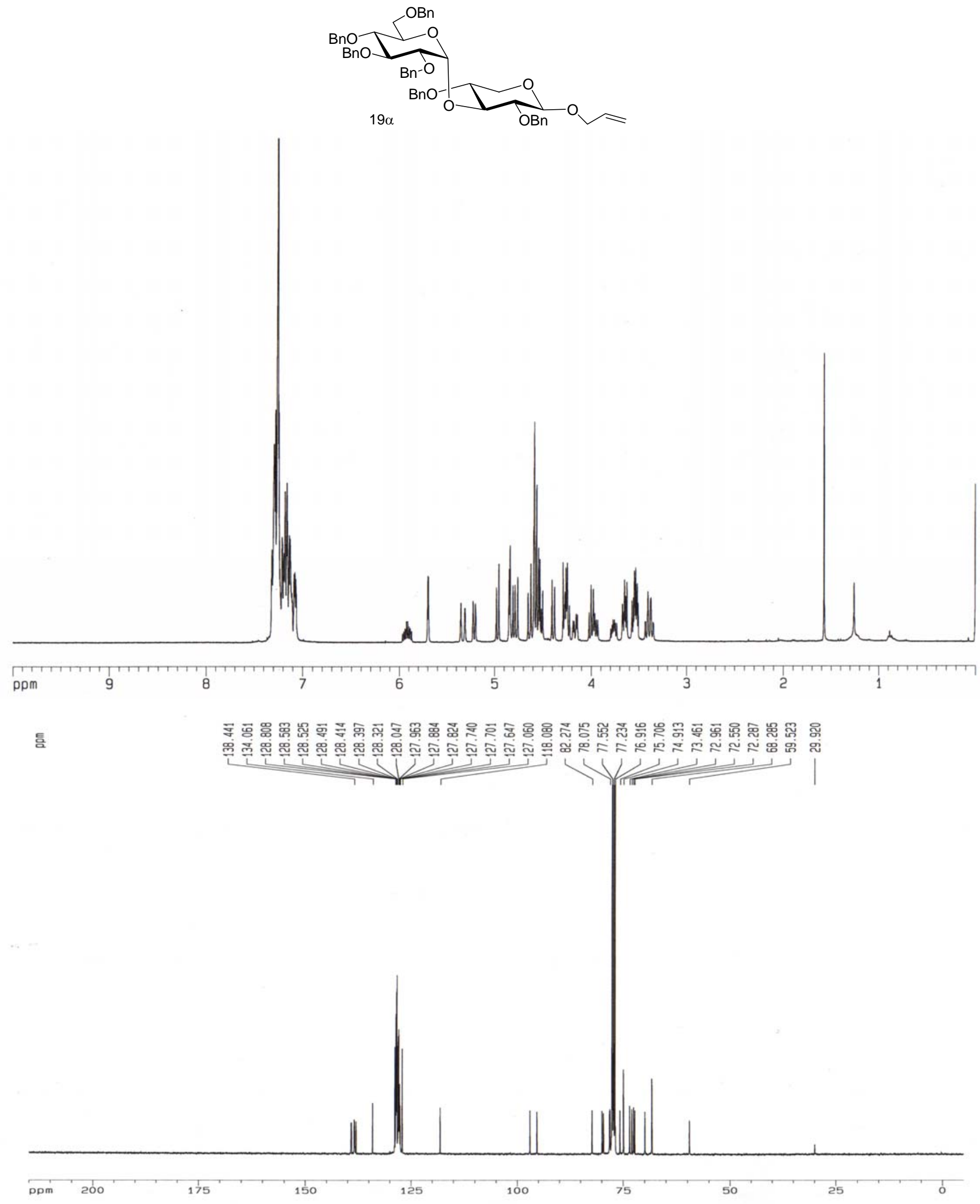



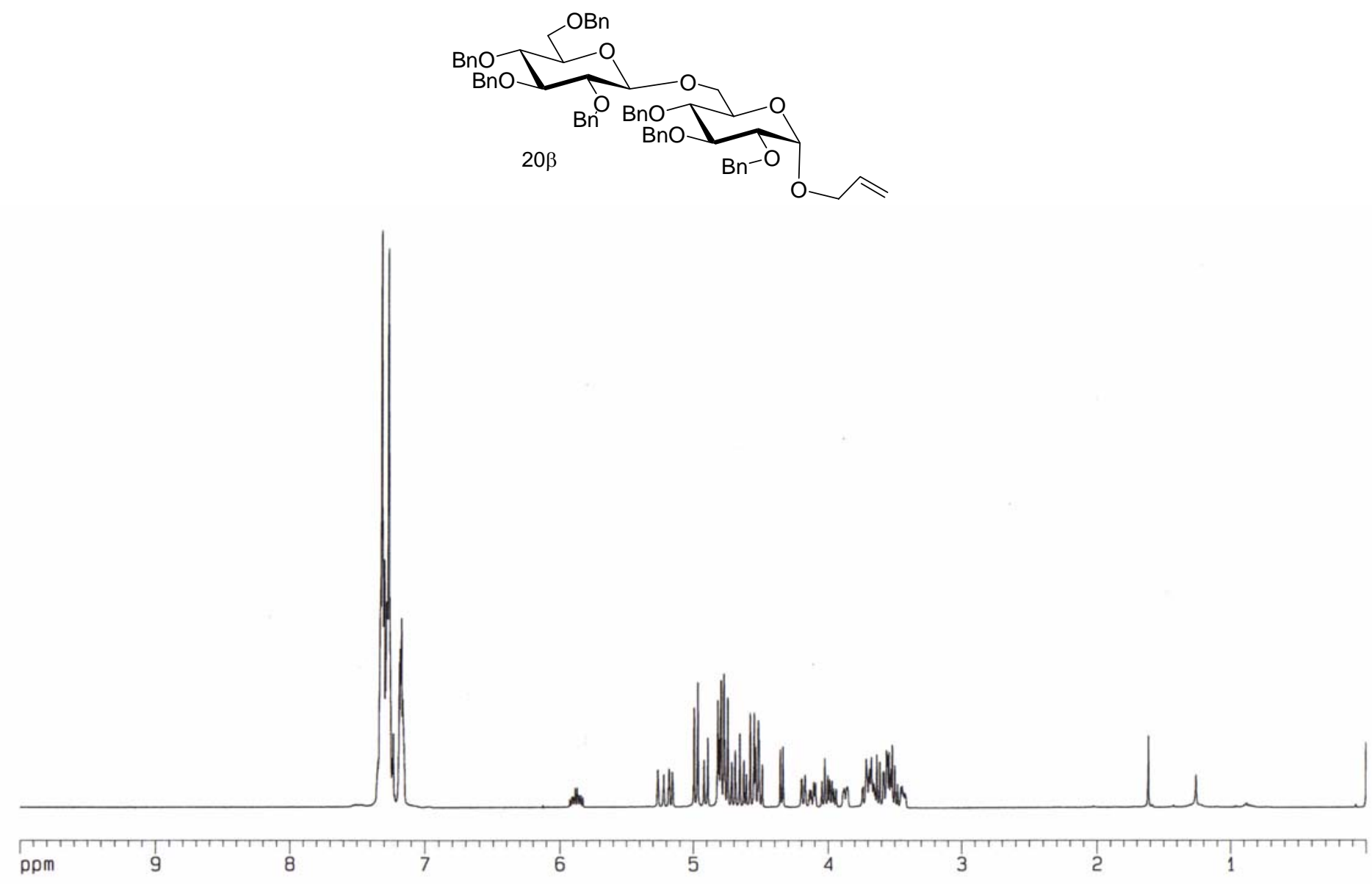

言

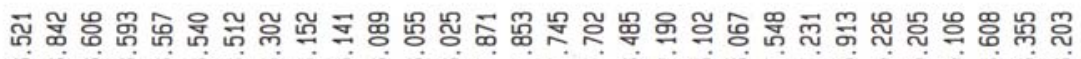

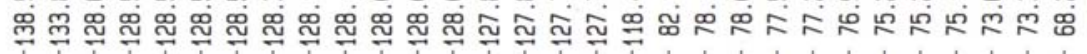

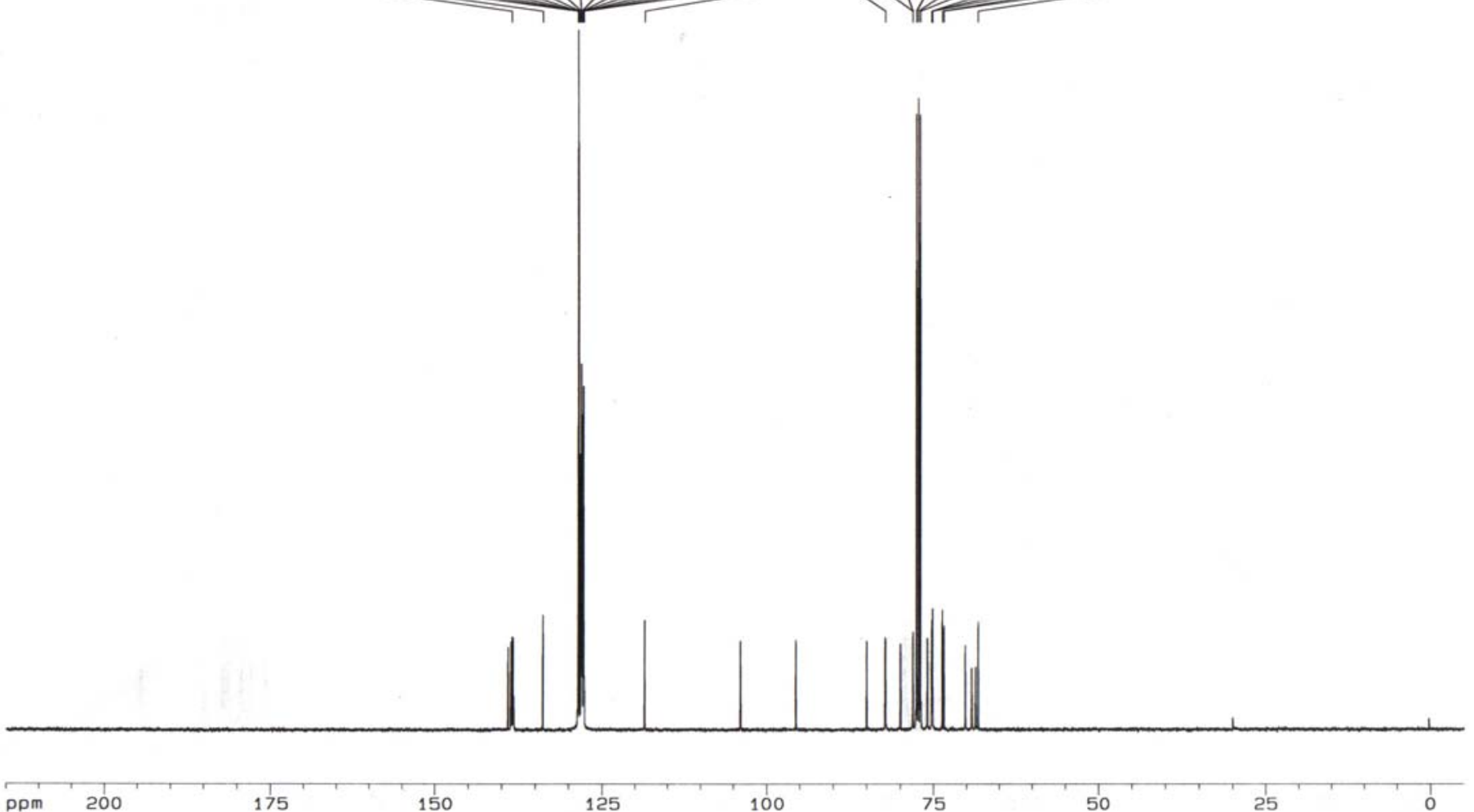

S-18 

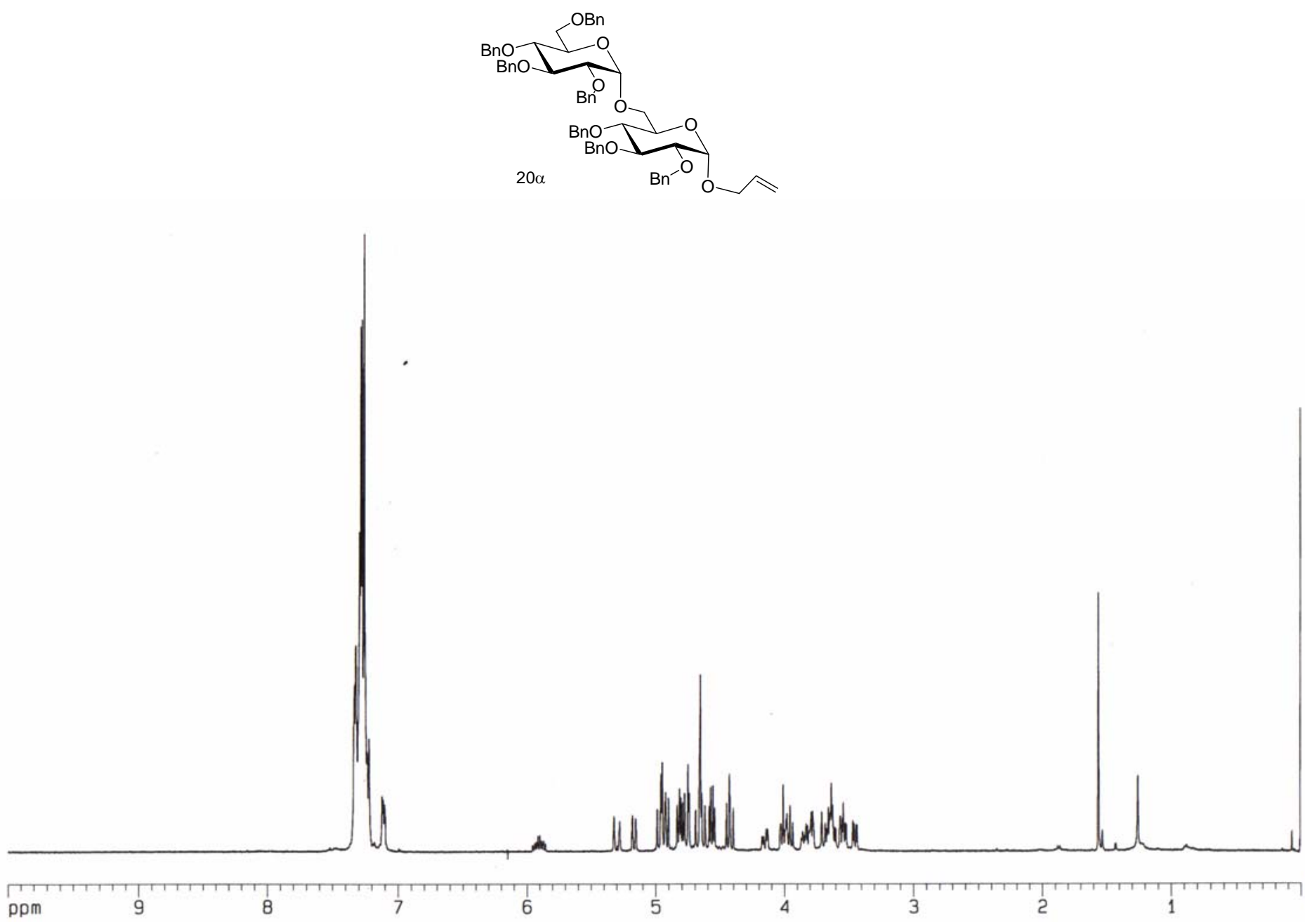

S-19 


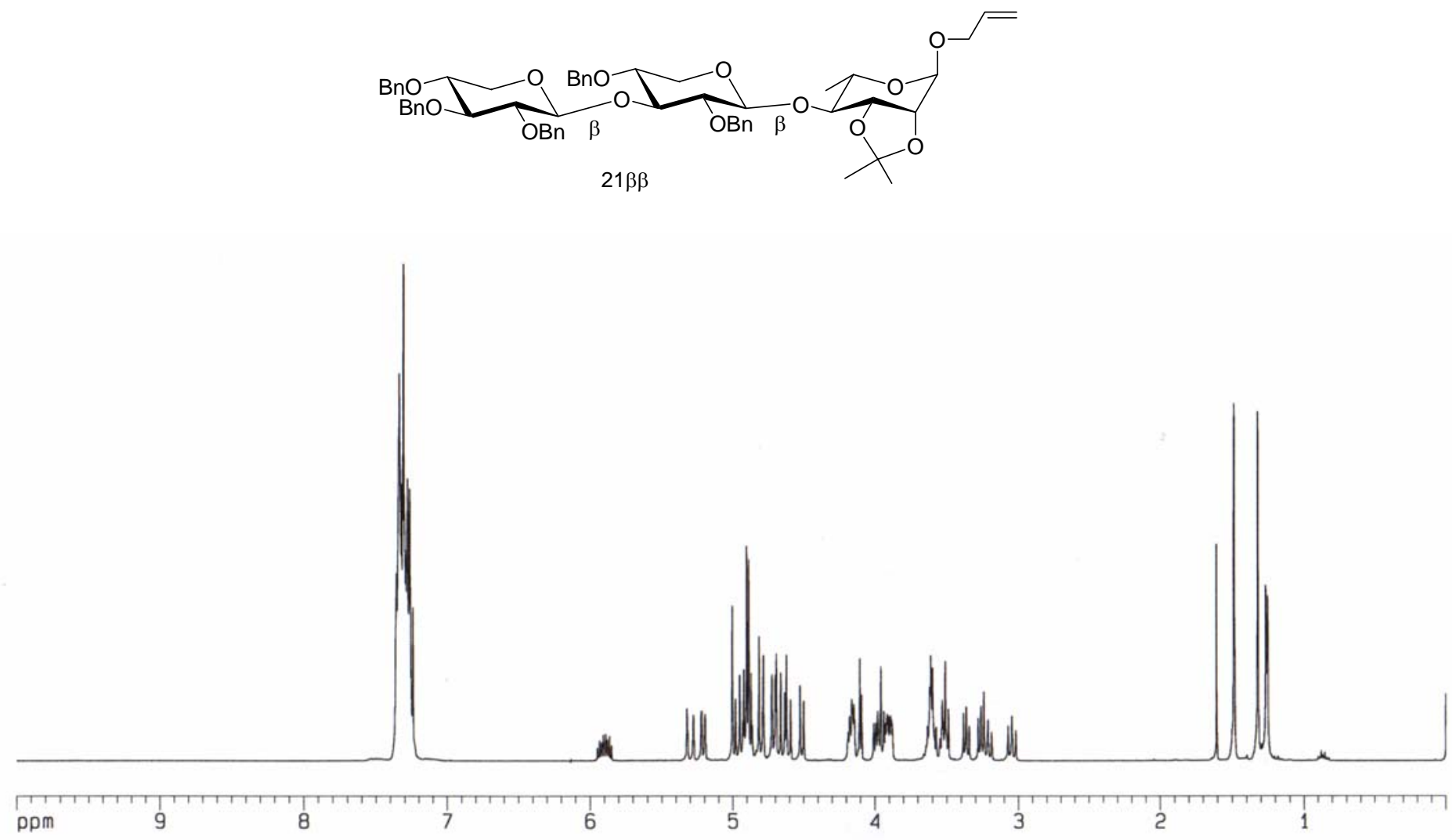

镸

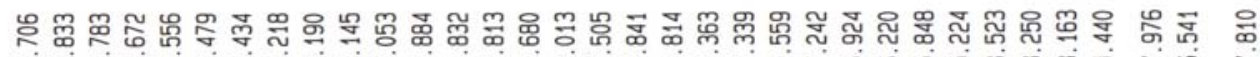

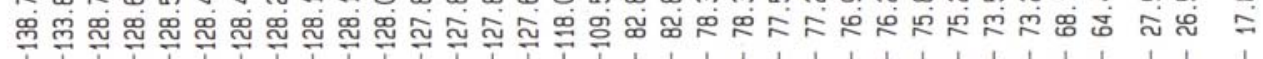
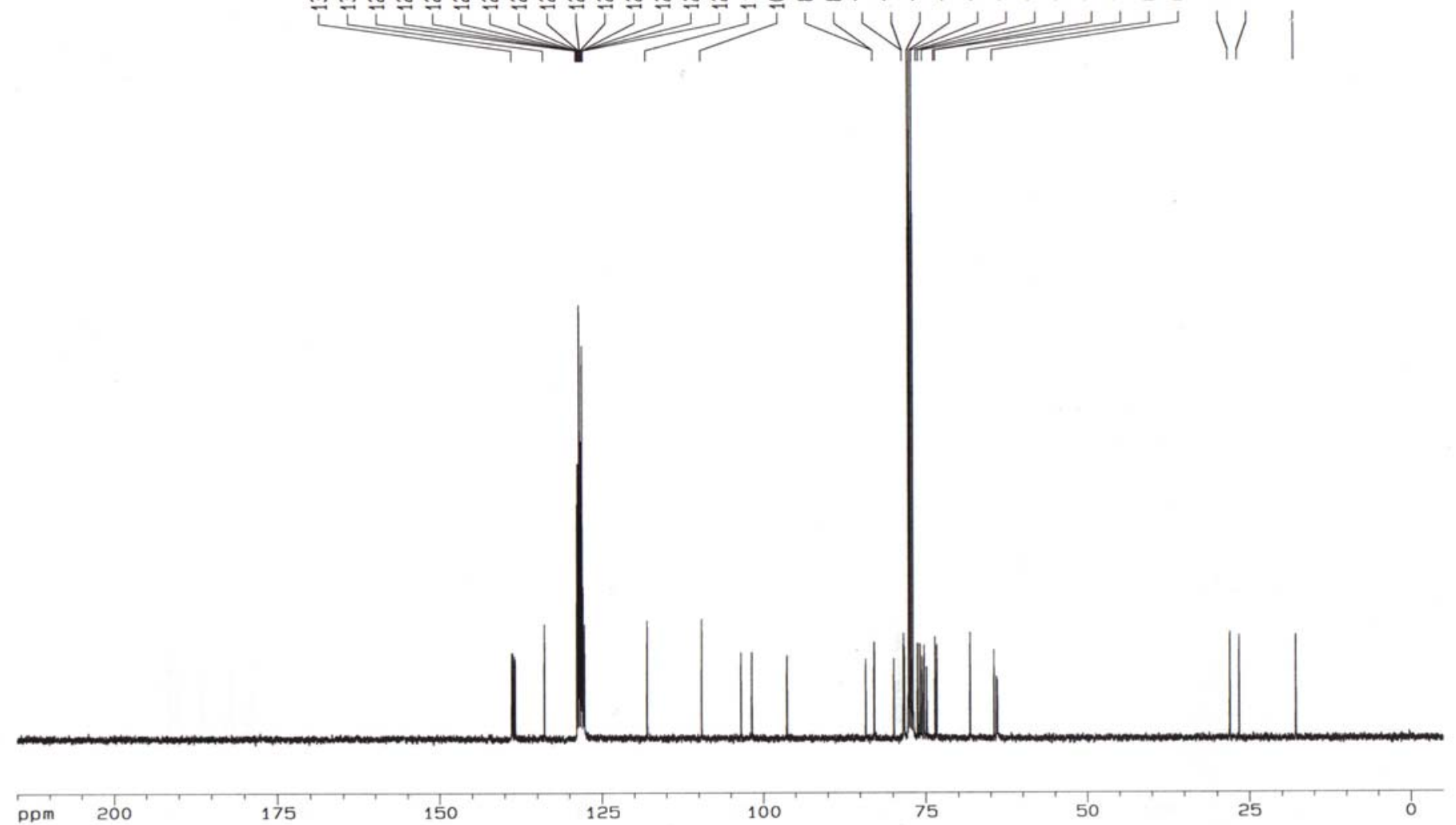

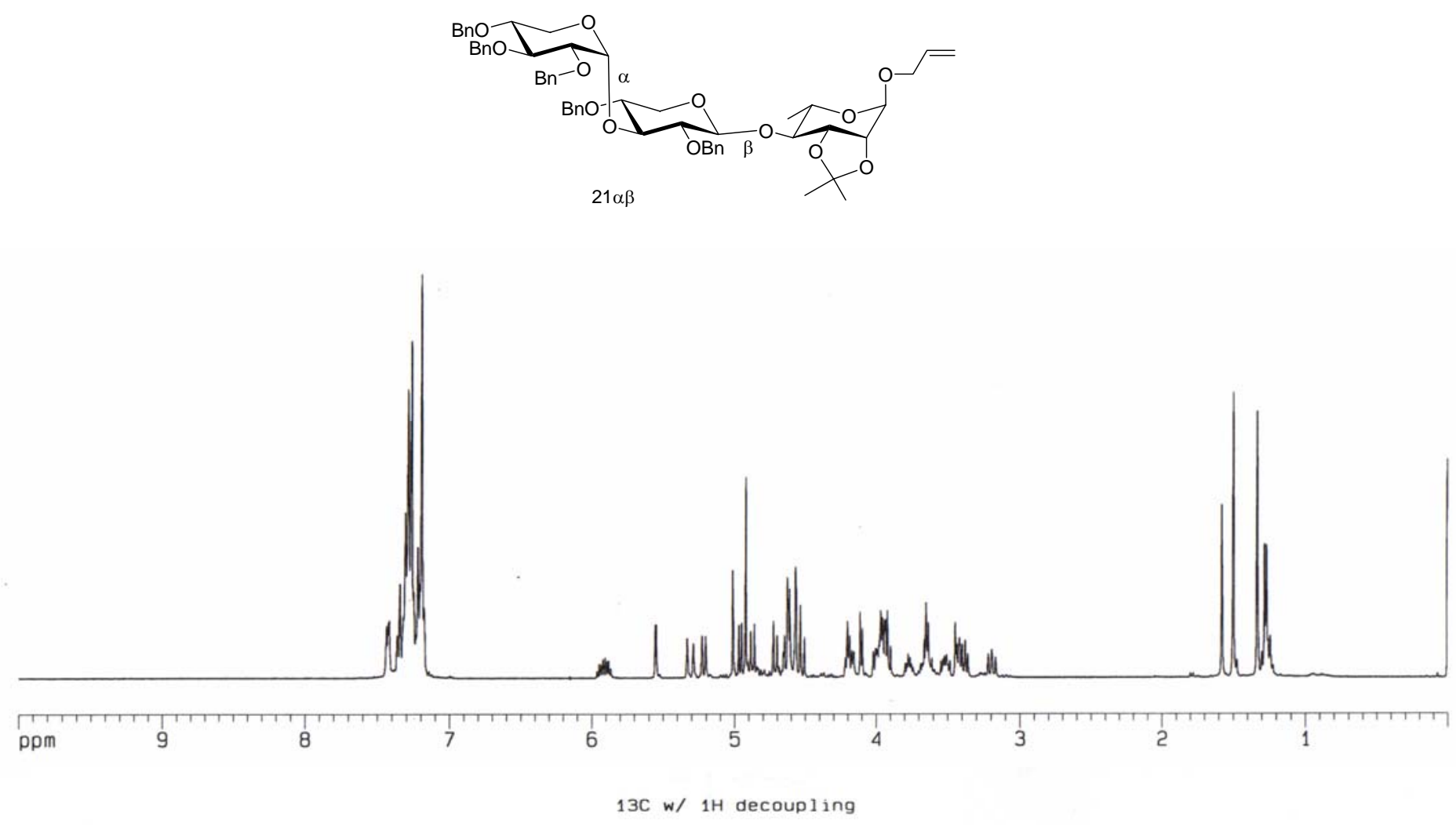

言

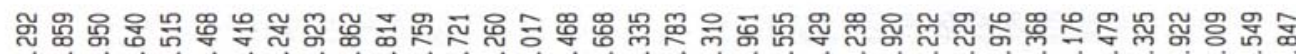

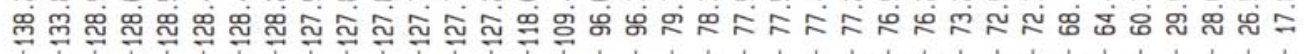

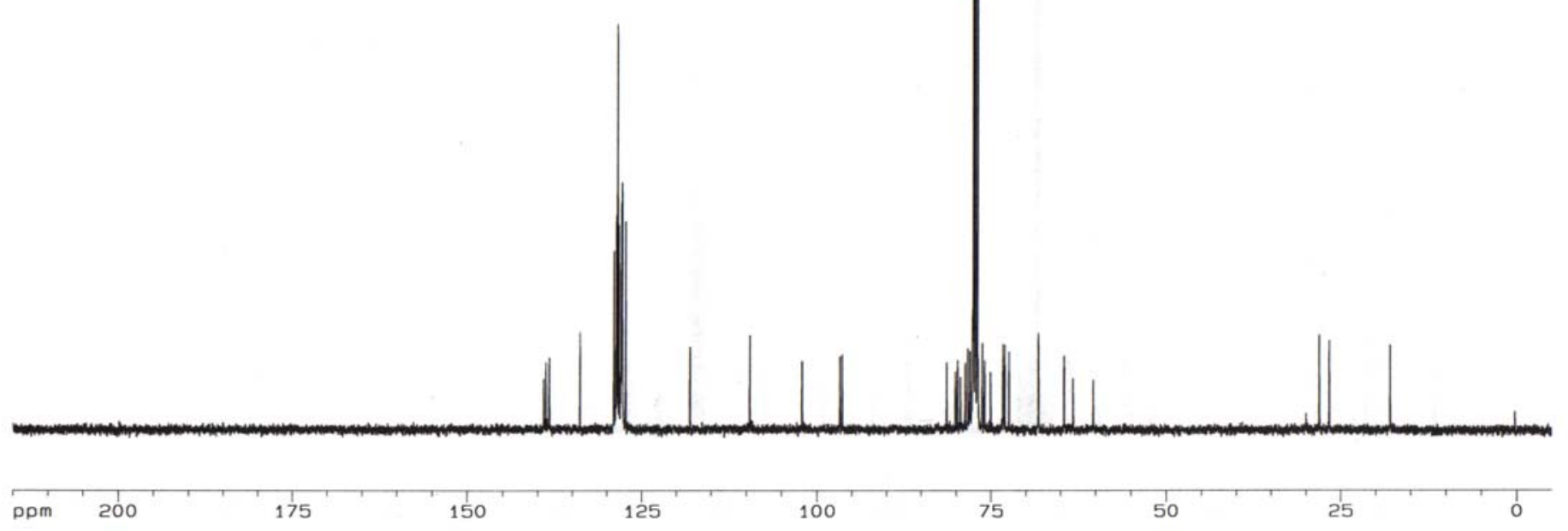




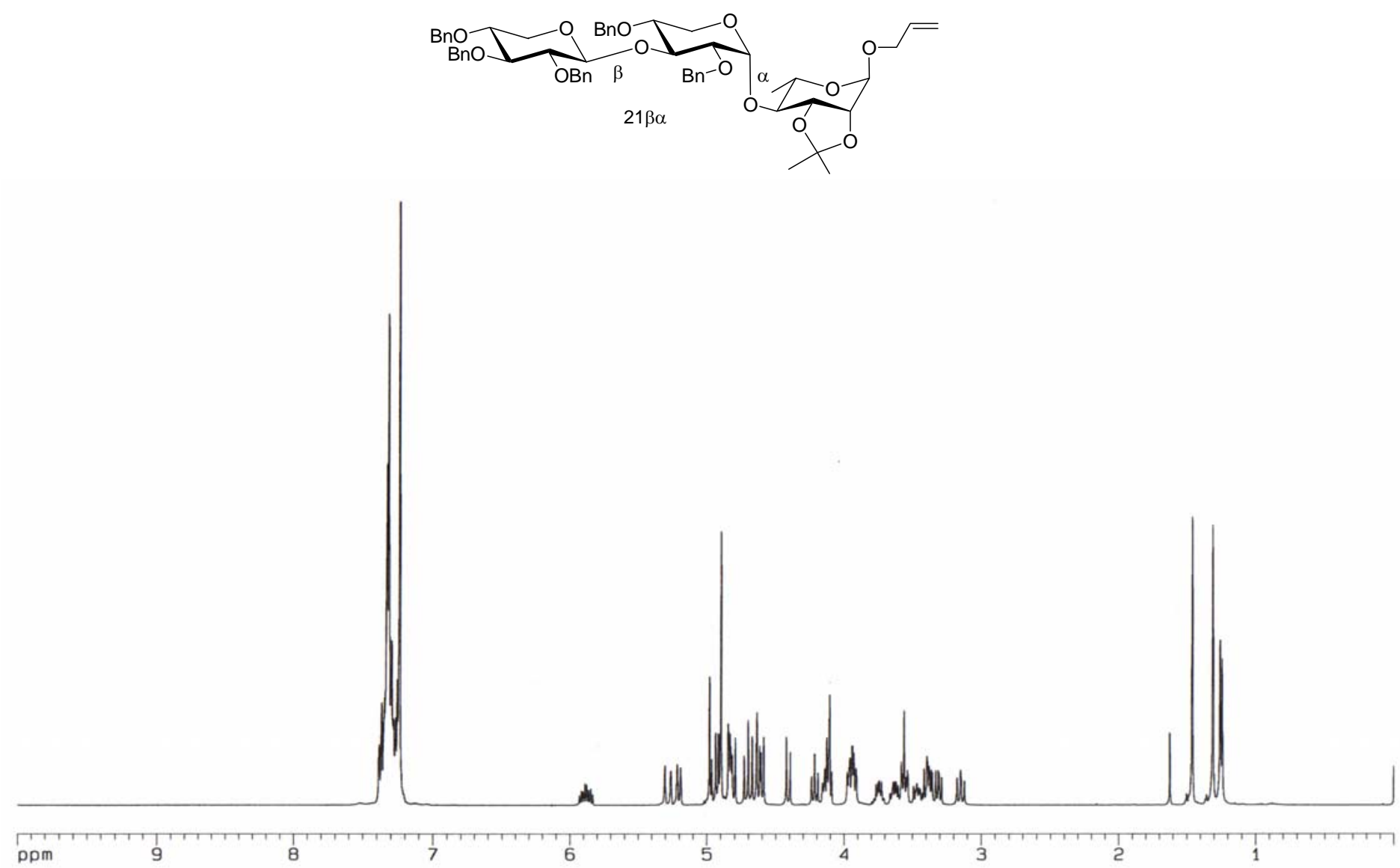

镸

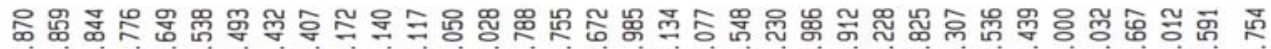

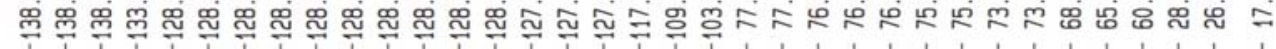
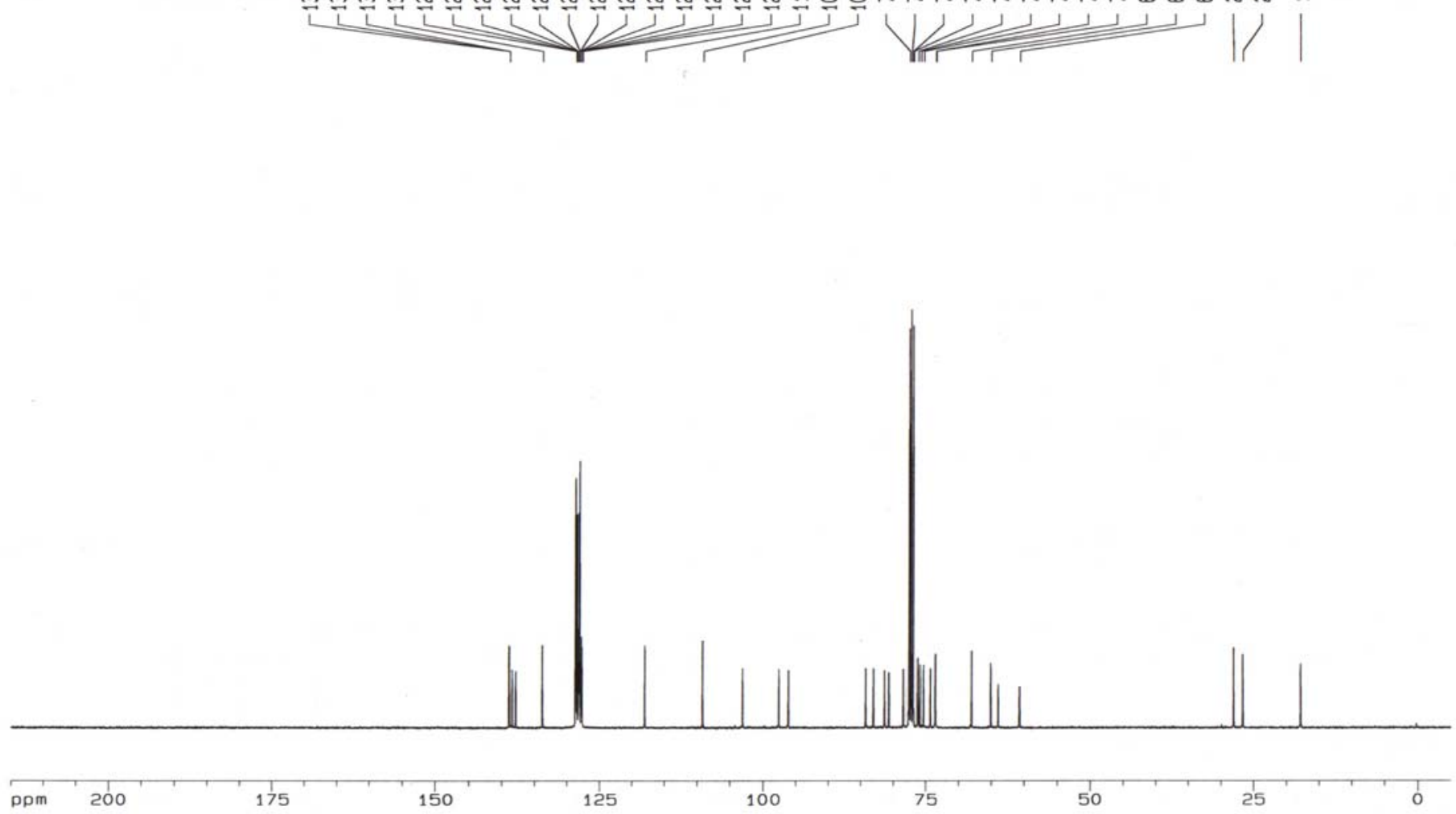


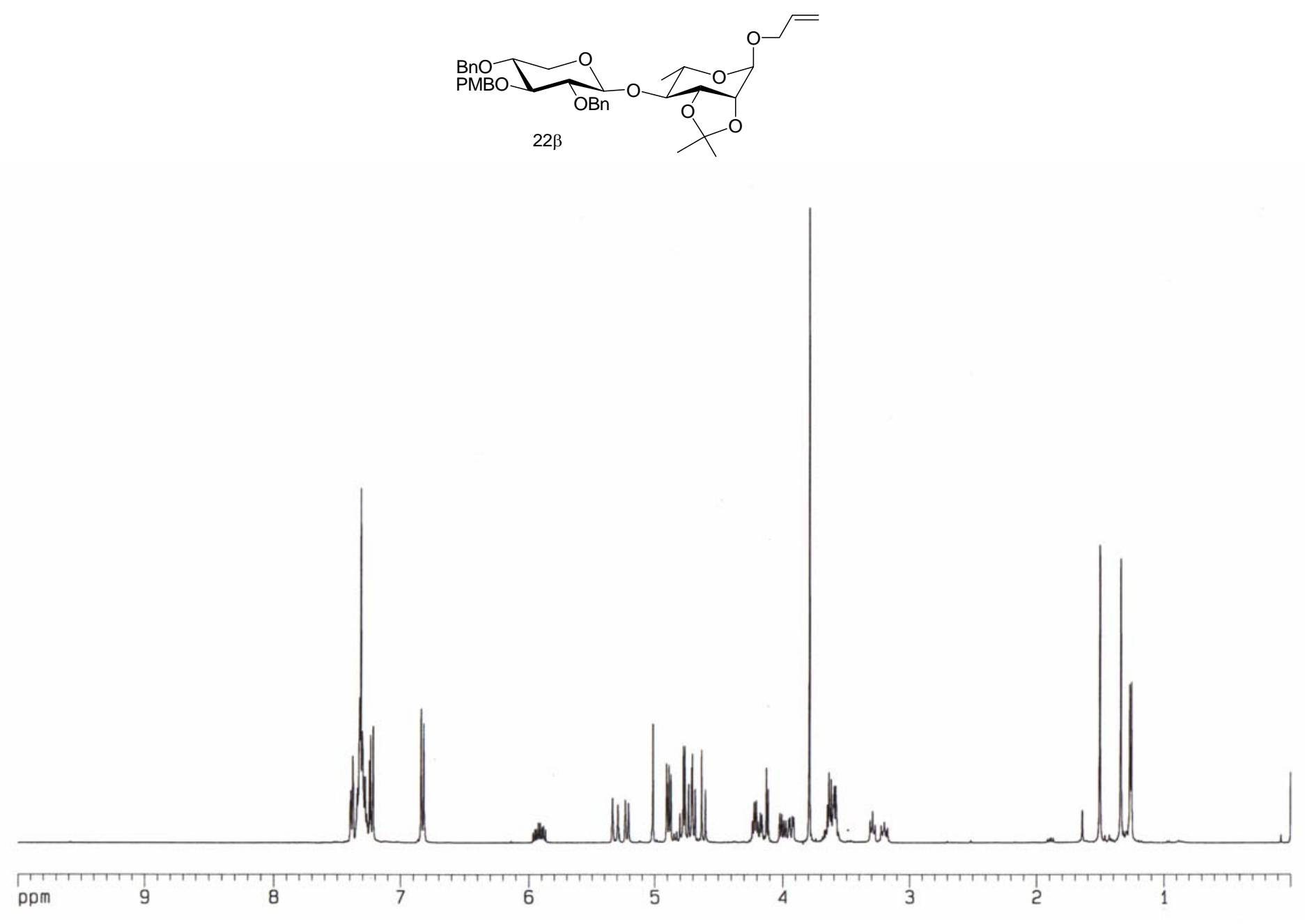

言

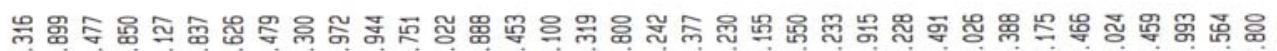

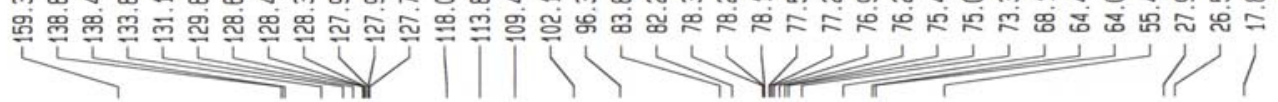

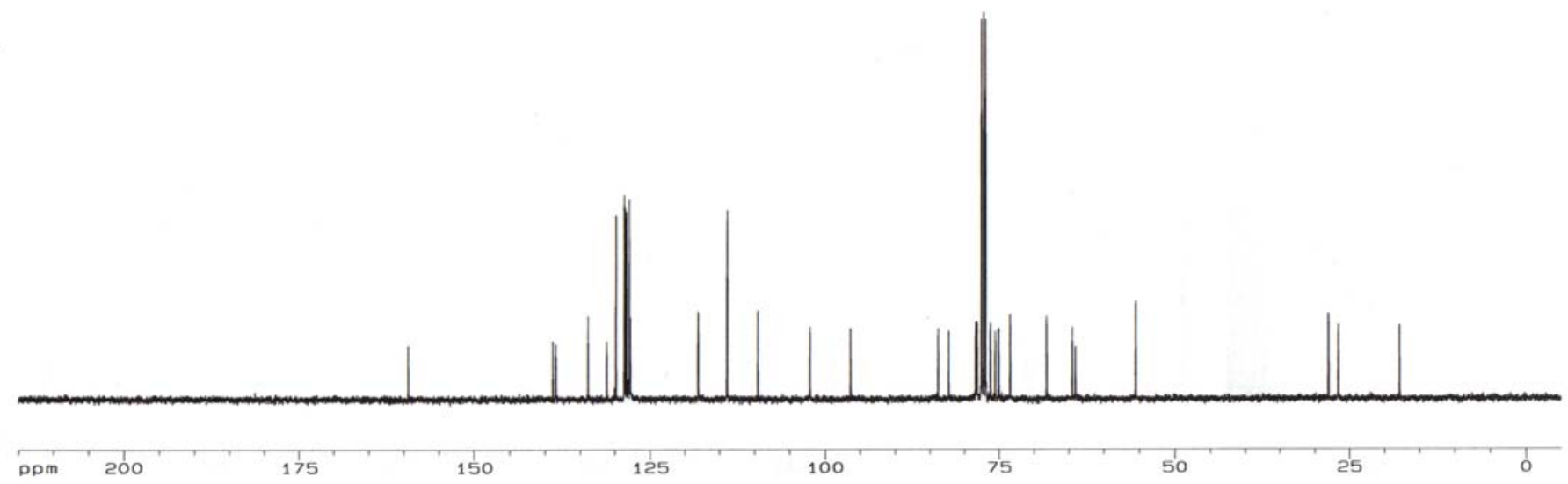




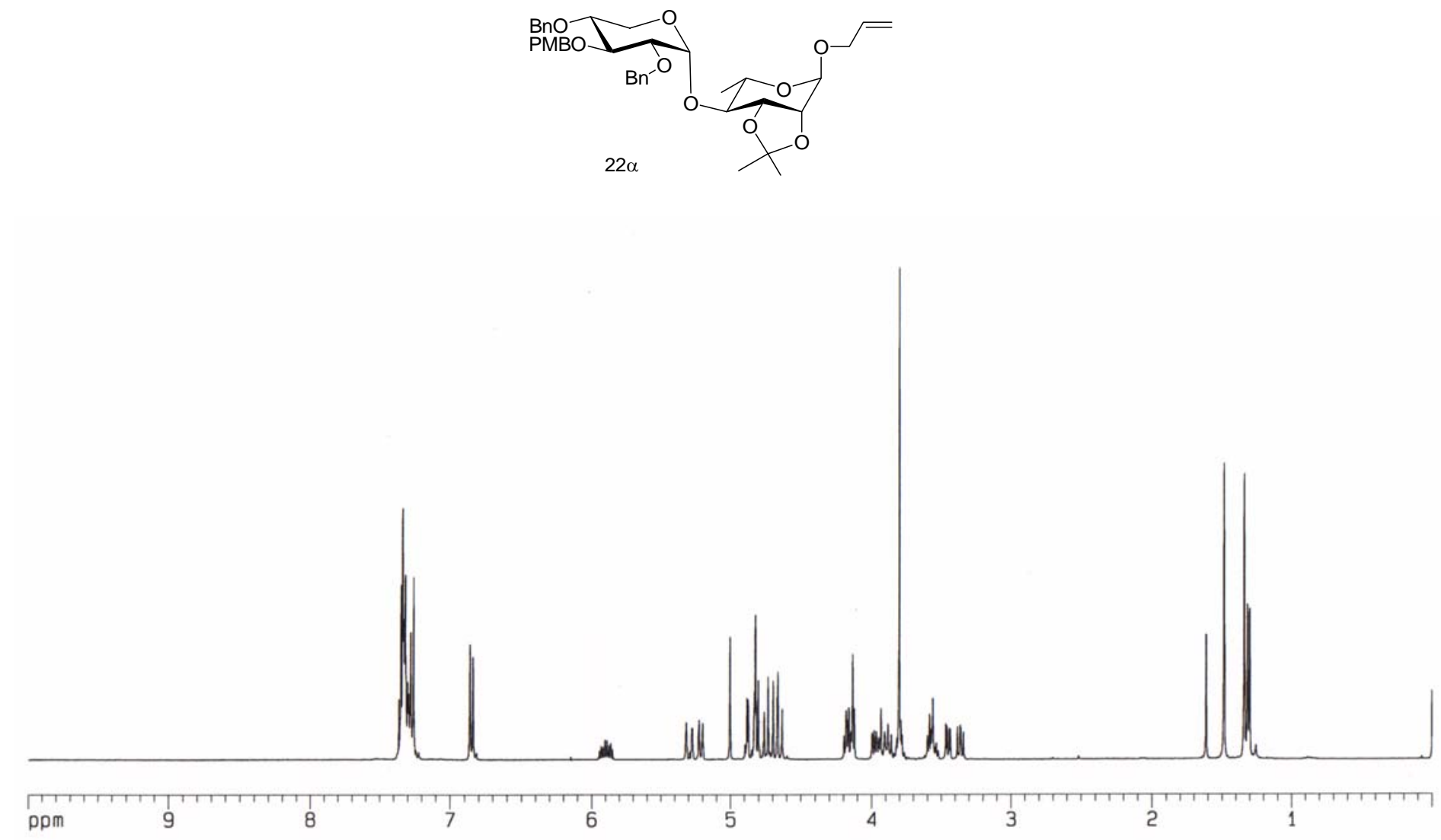

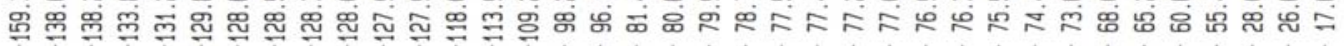
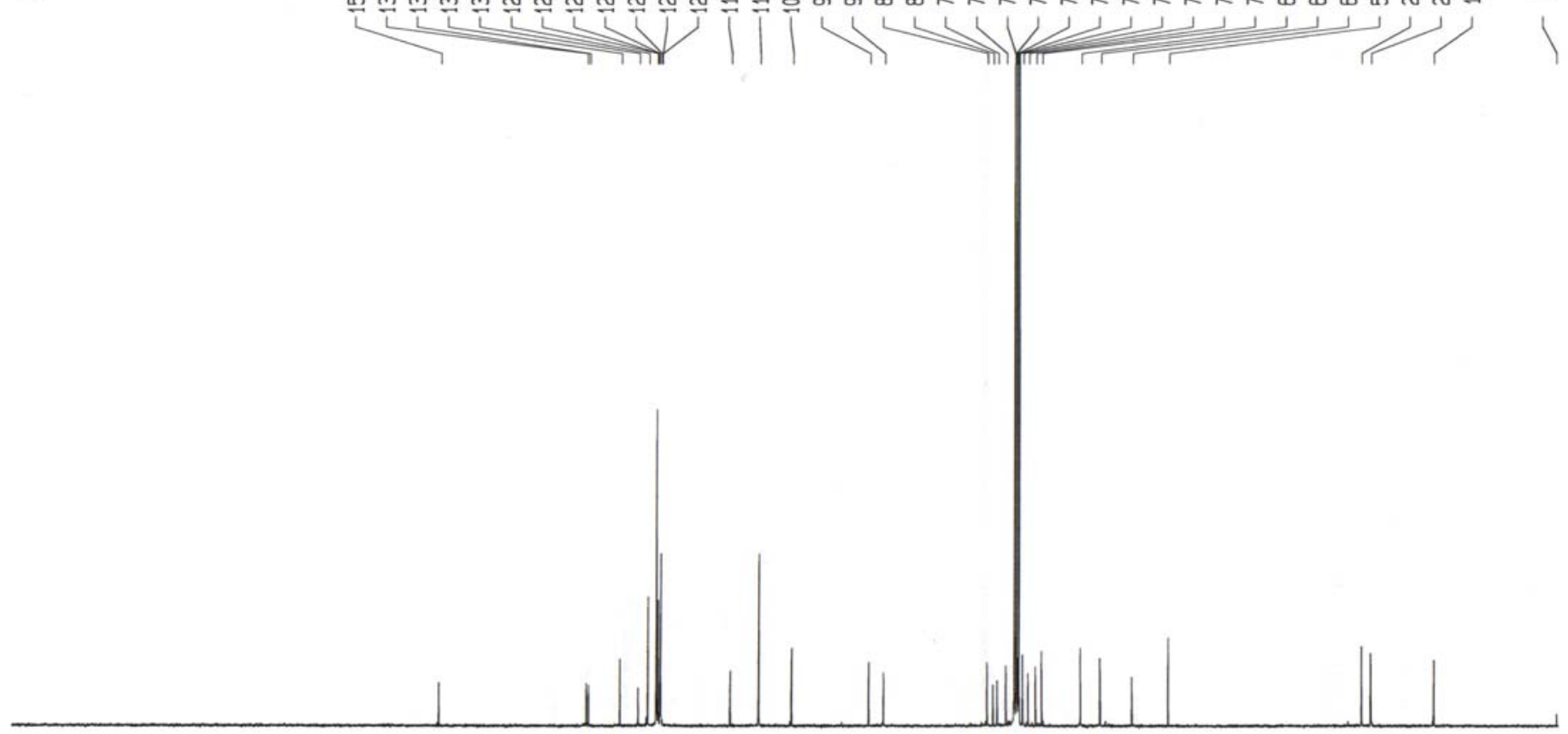


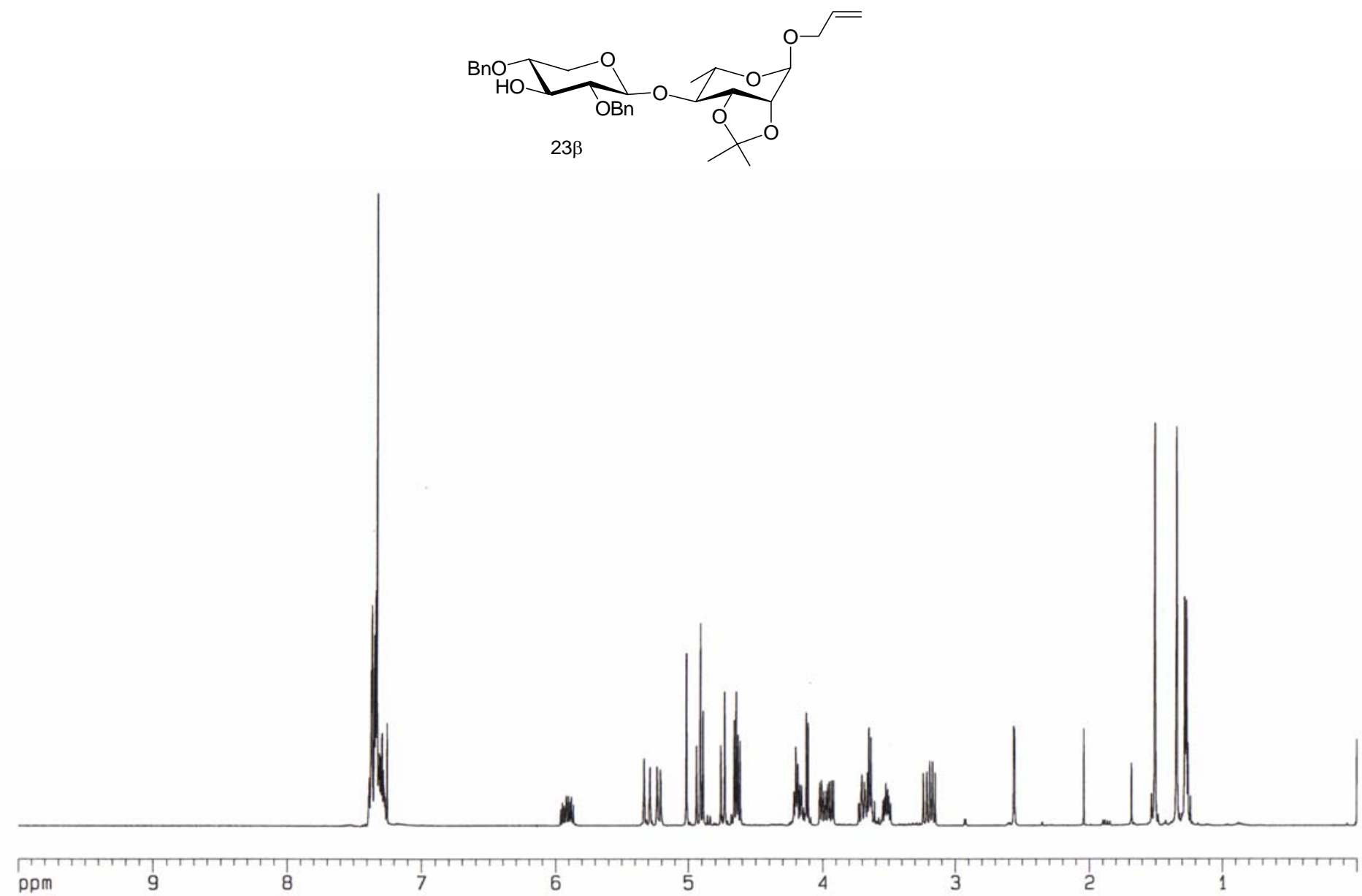

틈

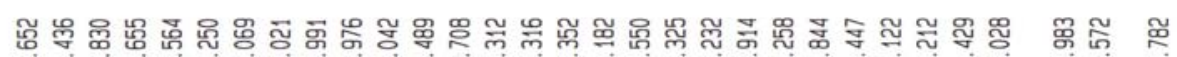

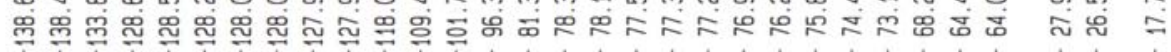

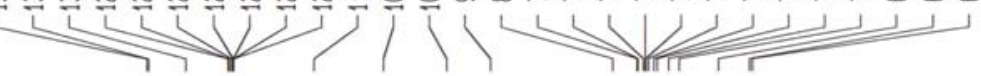
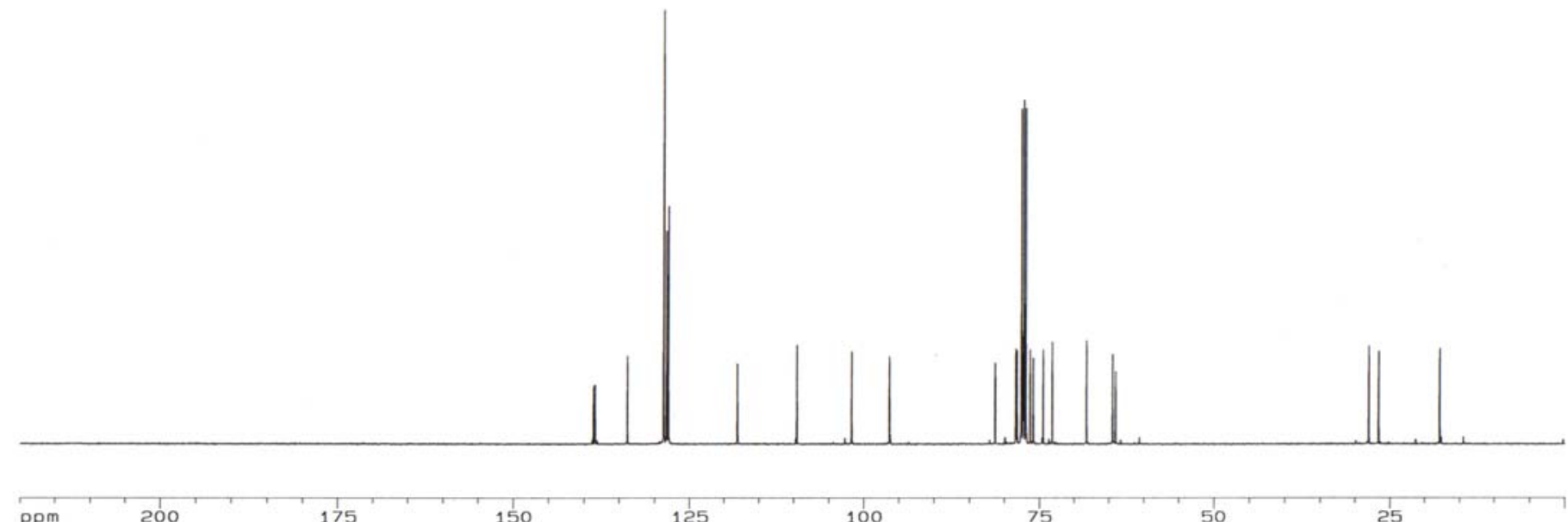

175

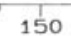

100

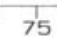

50

25 